\title{
Synchronization of two self-synchronous vibrating machines on an isolation frame
}

\author{
Chunyu Zhao ${ }^{\mathrm{a}, *}$, Qinghua Zhao ${ }^{\mathrm{b}}$, Zhaomin Gong ${ }^{\mathrm{a}}$ and Bangchun Wen ${ }^{\mathrm{a}}$ \\ ${ }^{a}$ School of Mechanical Engineering and Automation, Northeastern University, Shenyang 110004, China \\ ${ }^{\mathrm{b}}$ Hebei State-owned Minerals Development \& Investment Co., Ltd., Shijiazhuang, 050021, China
}

Received 10 February 2010

Revised 5 May 2010

\begin{abstract}
This paper investigates synchronization of two self-synchronous vibrating machines on an isolation rigid frame. Using the modified average method of small parameters, we deduce the non-dimensional coupling differential equations of the disturbance parameters for the angular velocities of the four unbalanced rotors. Then the stability problem of synchronization for the four unbalanced rotors is converted into the stability problems of two generalized systems. One is the generalized system of the angular velocity disturbance parameters for the four unbalanced rotors, and the other is the generalized system of three phase disturbance parameters. The condition of implementing synchronization is that the torque of frequency capture between each pair of the unbalanced rotors on a vibrating machine is greater than the absolute values of the output electromagnetic torque difference between each pair of motors, and that the torque of frequency capture between the two vibrating machines is greater than the absolute value of the output electromagnetic torque difference between the two pairs of motors on the two vibrating machines. The stability condition of synchronization of the two vibrating machines is that the inertia coupling matrix is definite positive, and that all the eigenvalues for the generalized system of three phase disturbance parameters have negative real parts. Computer simulations are carried out to verify the results of the theoretical investigation.
\end{abstract}

\section{Introduction}

In the last few decades, much effort has been devoted to mathematically explain the mechanism of synchronization. One of the early and most widely used approaches has been the method of direct separation of motions, i.e., that of two-timing (a special case of the method of multiple scales). Here the dynamics is divided into two parts: one corresponding to motion on a fast time scale, and the other on a slow time scale. Using the average Lagrange function of the vibrating system, Blekhman has applied this approach to successfully deal with a number of problems including problems of self-synchronization [2-8]. In vibrating systems with two identical unbalanced rotors, the approach is greatly simplified by combining the differential equations of the two unbalanced rotors into the differential equation of the phase difference between the two unbalanced rotors $[14,15]$. Using the Hill equations and the Floquet theory, Yamapi and Woafo derived instability and the complete synchronization in the ring of four coupled self-sustained electromechanical devices $[16,17]$. Taking the two disturbance parameters of the average angular velocity of the two unbalanced rotors in a vibrating system as the small parameters, the authors deduced the non-dimensional coupling equations of the rotors. The stability for synchronization of two unbalanced rotors is converted into the problem of stability for a system of the three first order differential equations and the stability condition is derived by means of the Routh-Hurwitz criterion [18,19] or a general Lyapunov function [20]. On the other hands, several numeric methods have been developed to tackle the problem of synchronization $[1,9,10,12,13]$. But when the number of the unbalanced rotors is more than two, investigation of the stability is very difficult with the above methods.

\footnotetext{
*Corresponding author. E-mail: chyzhao@mail.neu.edu.cn.
} 


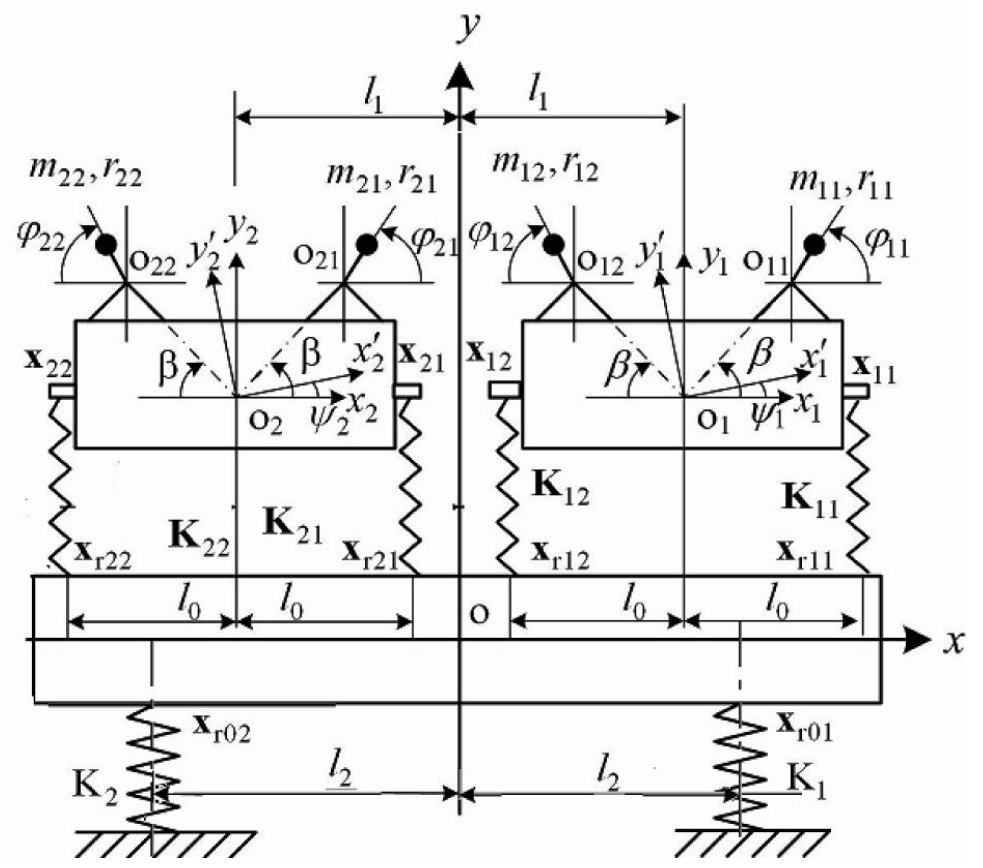

Fig. 1. Dynamic model of a vibration isolation system.

Taking two self-synchronous vibrating machines on an isolation rigid frame for example, this paper extends our previous works on the synchronization of two unbalanced rotors into the synchronization of multiple unbalanced rotors in a vibrating system. Herein, the problem of synchronization stability for multiple unbalanced rotors is divided into that of two generalized systems. One is the generalized system for the disturbance parameters of multiple angular velocities, and the other is the first order differential equations for the disturbance parameters of phase differences whose number is less than the number of unbalanced rotors by one. In the next section, the equations of motion of the system are described. The condition of implementing synchronization and that of stability of synchronization are deduced in Section 3. Computer simulations are carried out to verify the theoretical results in Section 4. Finally, conclusions are provided in Section 5.

\section{Equations of motion}

Figure 1 shows the dynamic model of a vibration isolation system, which consists of two vibrating machines, denoted by V1 and V2, and an isolation rigid frame. The rigid frame is supported by an elastic foundation, which is composed of the two groups of springs installed symmetrically. Each vibrating machine is supported on the rigid frame by two groups of springs installed symmetrically and excited by two unbalanced rotors, which are separately driven by two induction motors rotating in opposite directions.

As illustrated in Fig. 1, the projection $o$ of the mass center $G$ of the rigid frame onto the $y$-axis is fixed and that of the mass center $G_{i}$ of the mass center of the vibrating machine $\mathrm{V} i$ onto the $y_{i}$-axis is also fixed. For the rigid frame, three reference frames are assigned: the fixed frame oxy with the $y$-axis vertical and coinciding with the center line of the rigid frame; the nonrotating moving frame $G x^{\prime} y^{\prime} z^{\prime}$, which undergoes translation motions while remaining parallel to $o x y$, and the moving frame $G x^{\prime \prime} y^{\prime \prime}$ that is fixed to the rigid frame, as shown in Fig. 2(a). For each vibrating machine, three reference frames also are assigned: the fixed frame $o_{i} x_{i} y_{i}$, the nonrotating moving frame $G x_{i}^{\prime} y_{i}^{\prime} z_{i}^{\prime}$ and the moving frame $G_{i} x_{i}^{\prime \prime} y_{i}^{\prime \prime}, i=1,2$, as shown in Fig. 2(b).

Because the rigid frame is supported by the elastic foundation, it exhibits three degrees of freedoms. Mass center coordinates, $x$ and $y$, and an angular rotation $\psi$ are set as independent coordinates. In like manner, the mass center coordinates of vibrating machine $\mathrm{V}_{i}, x_{i}$ and $y_{i}$, and its angular rotation $\psi_{i}$ also are set as independent coordinates. 

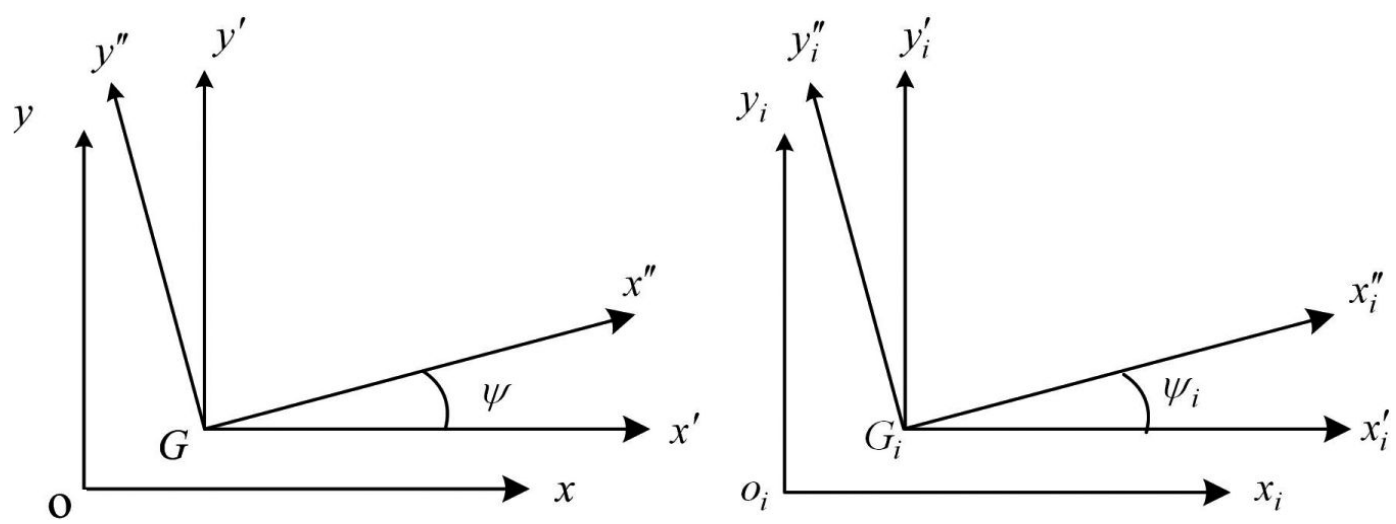

Fig. 2. Reference frames: the fixed frame oxyand $o_{i} x_{i} y_{i} ; G y^{\prime} z^{\prime}$ and $G_{i} y_{i}^{\prime} z_{i}^{\prime}$ nonrotating moving frame; $G y^{\prime \prime} z^{\prime \prime}, G_{i} y_{i}^{\prime \prime} z_{i}^{\prime \prime}$ and moving frame fixed to the rigid frame $i$.

In the reference frame $G_{i} x_{i}^{\prime \prime} y_{i}^{\prime \prime}$, the coordinates of mass center of each eccentric lump can be expressed as

$$
\boldsymbol{x}^{\prime \prime}{ }_{m i 1}=\left\{\begin{array}{l}
l_{a i} \cos \beta+r_{i 1} \cos \varphi_{i 1} \\
l_{a i} \sin \beta+r_{i 1} \sin \varphi_{i 1}
\end{array}\right\}, \quad \boldsymbol{x}^{\prime \prime}{ }_{m i 2}=\left\{\begin{array}{c}
-l_{a i} \cos \beta-r_{i 1} \cos \varphi_{i 2} \\
l_{a i} \sin \beta+r_{i 1} \sin \varphi_{i 2}
\end{array}\right\}(i=1,2 .)
$$

where $l_{a i}$ is the distance between the mass center of vibrating machine Vi and the rotating center of the eccentric lump; and $\beta$ is the angle between the line $o_{i} o_{i j}$ and the $x$-axis.

In the reference frame $G_{i} x_{i} y_{i}$, the coordinates of the eccentric lump can be expressed as the following

$$
\boldsymbol{x}_{m i j}=\boldsymbol{x}_{G i}+\boldsymbol{R}_{i} \boldsymbol{x}_{\mathrm{m} i j}, \boldsymbol{R}_{i}=\left[\begin{array}{l}
\cos \psi_{i}-\sin \psi_{i} \\
\sin \psi_{i} \cos \psi_{i}
\end{array}\right](i=1,2, ; j=1,2 .)
$$

where $\boldsymbol{x}_{G i}$ is the coordinate vector of the mass center $G_{i}$ of the vibrating machine Vi, $\boldsymbol{x}_{G i}=\left\{x_{i}, y_{i}\right\}^{T}$.

Then the kinetic energy $T$ of the system is expressed as

$$
\begin{aligned}
T= & \frac{1}{2} \dot{\boldsymbol{x}}_{G}^{T} \boldsymbol{m}_{R} \dot{\boldsymbol{x}}_{G}+\frac{1}{2} J_{R} \dot{\psi}^{2}+\frac{1}{2} \sum_{i=1}^{2} \dot{\boldsymbol{x}}_{G i} \boldsymbol{m}_{i} \dot{\boldsymbol{x}}_{G i}+\frac{1}{2} \sum_{i=1}^{2} J_{i} \dot{\psi}_{i}^{2}+ \\
& \frac{1}{2} \sum_{i=1}^{2} \sum_{j=1}^{2} \boldsymbol{x}_{m i j}^{T} \boldsymbol{m}_{i j} \boldsymbol{x}_{m i j}+\frac{1}{2} \sum_{i=1}^{2} \sum_{j=1}^{2} J_{0 i j} \dot{\varphi}_{i j}^{2}
\end{aligned}
$$

where $\boldsymbol{m}_{R}$ is the mass matrix of the isolation frame, $\boldsymbol{m}_{R}=\operatorname{diag}\left(m_{R}, m_{R}\right)$, and $J_{R}$ is its moment of inertia about its mass center; $\boldsymbol{x}_{G}$ is the displacement vector of the isolation frame, $\boldsymbol{x}_{G}=\{x, y\}^{T} ; \boldsymbol{m}_{i}$ is the mass matrix of the vibrating machine $\mathrm{Vi}, \boldsymbol{m}_{i}=\operatorname{diag}\left(m_{V}, m_{V}\right)$, and $J_{i}$ is its moment of inertia about its mass center; $\boldsymbol{m}_{i j}$ is the mass matrix of the eccentric lump in the vibrating machine $\mathrm{Vi}$ and $J_{0 i j}$ is the moment of inertia of motor $j$ in the vibrating machine $\mathrm{Vi} ;(\bullet)$ denotes $\mathrm{d} \bullet / \mathrm{d} t$.

When the vibrating system is running, the coordinates of the points that the supporting springs of the rigid frame are connected to the rigid frame on the reference oxy can be expressed as

$$
\boldsymbol{x}_{r i}=\boldsymbol{x}_{o}+\boldsymbol{R}_{r 0 i}, \quad \boldsymbol{R}=\left[\begin{array}{l}
\cos \psi-\sin \psi \\
\sin \psi \cos \psi
\end{array}\right], \quad i=1,2 .
$$

where $\boldsymbol{x}_{o}$ is the displacement vector of the mass center of the rigid frame, $\boldsymbol{x}_{o}=\{x, y\}^{T} ; \boldsymbol{x}_{r 01}$ and $\boldsymbol{x}_{r 02}$ are the coordinates of the corresponding points on the reference frame oxy when the system is in the equilibrium state, $\boldsymbol{x}_{r 01} \approx\left\{l_{k}, 0\right\}^{T}$ and $\boldsymbol{x}_{r 02} \approx\left\{-l_{k}, 0\right\}^{T}$.

Then, the vector of transformation of the corresponding spring can be expressed as

$$
\Delta \boldsymbol{x}_{r i}=\boldsymbol{x}_{r i}-\boldsymbol{x}_{r 0 i}, \quad i=1,2 .
$$


The coordinates of point of the supporting spring of the vibrating machine $\mathrm{V} i, \mathrm{X}_{i j}$, connected to itself on the reference frame $o_{i} x_{i} y_{i}$, are $\boldsymbol{x}_{i j}$, then we can obtain its coordinate on $o_{i} x_{i} y_{i}$-frame as the following

$$
\boldsymbol{x}_{i j}^{\prime}=\boldsymbol{x}_{o i}+\boldsymbol{R}_{i} \boldsymbol{x}_{i j}, \quad \boldsymbol{R}_{i}=\left[\begin{array}{l}
\cos \psi_{i}-\sin \psi_{i} \\
\sin \psi_{i} \cos \psi_{i}
\end{array}\right](i=1,2 ; j=1,2 .)
$$

Assuming that the coordinates of point of the supporting spring of vibrating machine $\mathrm{V} i, \mathrm{~K} i$, connected to the main rigid frame is $\boldsymbol{x}_{r i j}$ on the reference frame oxy, then, during the operation of the system, its coordinates on the reference frame oxy can be expressed as

$$
\boldsymbol{x}_{r i j}^{\prime}=\boldsymbol{x}_{o}+\boldsymbol{R} \boldsymbol{x}_{r i j}(i=1,2 ; j=1,2 .)
$$

and the transformation vector of spring $\mathrm{K}_{i j}$ is expressed as

$$
\Delta \boldsymbol{x}_{i j}=\left(\boldsymbol{x}_{o i}-\boldsymbol{x}_{o}\right)+\left(\boldsymbol{R}_{i}-\boldsymbol{I}\right) \boldsymbol{x}_{i j}-(\boldsymbol{R}-\boldsymbol{I}) \boldsymbol{x}_{r i j}
$$

Then the potential energy $V$ of the system is expressed as

$$
V=\frac{1}{2} \sum_{i=1}^{2} \Delta \boldsymbol{x}_{r i}^{T} \boldsymbol{K}_{i} \Delta \boldsymbol{x}_{r i}+\frac{1}{2} \sum_{i=1}^{2} \sum_{j=1}^{2} \Delta x_{i j}^{T} \boldsymbol{K}_{i j} \Delta \boldsymbol{x}_{i j}
$$

where $\boldsymbol{K}_{i}$ is the stiffness matrix of the supporting spring $i$ of the rigid frame, $\boldsymbol{K}_{i}=\operatorname{diag}\left(K_{x} / 2, K_{y} / 2\right)$; and $\boldsymbol{K}_{i j}$ is the stiffness matrix of the supporting spring $j$ of vibrating machine $\mathrm{V} i, \boldsymbol{K}_{i j}=\operatorname{diag}\left(k_{x} / 2, k_{y} / 2\right)$.

The viscous dissipation function $D$ of the system can be described as the following:

$$
D=\frac{1}{2} \sum_{i=1}^{2} \Delta \dot{\boldsymbol{x}}_{i}^{T} \boldsymbol{F}_{i} \Delta \dot{\boldsymbol{x}}_{i}+\frac{1}{2} \sum_{i=1}^{2} \sum_{j=1}^{2} \Delta \dot{\boldsymbol{x}}_{i j}^{T} \boldsymbol{F}_{i j} \Delta \dot{\boldsymbol{x}}_{i j}+\frac{1}{2} \sum_{i=1}^{2} \sum_{j=1}^{2} f_{j i} \dot{\varphi}_{i j}^{2}
$$

where $\boldsymbol{F}_{i}$ is the damping matrix of the supporting spring $i$ of the rigid frame, $\boldsymbol{F}_{i}=\operatorname{diag}\left(F_{x} / 2, F_{y} / 2\right)$; and $\boldsymbol{F}_{i j}$ is the stiffness damping of the supporting spring $j$ of vibrating machine $\mathbf{V} i, \boldsymbol{F}_{i j}=\operatorname{diag}\left(f_{x} / 2, f_{y} / 2\right)$.

The equations of motion are set up by using Lagrange's equations

$$
\frac{\mathrm{d}}{\mathrm{d} t} \frac{\partial(T-V)}{\partial \dot{q}_{i}}-\frac{\partial(T-V)}{\partial q_{i}}+\frac{\partial D}{\partial \dot{q}_{i}}=Q_{i}
$$

where $q_{i}$ is the generalized coordinates of the considered system and $Q_{i}$ is the system generalized force.

If $\boldsymbol{q}=\left[x, y, \psi, x_{1}, y_{1}, \psi_{1}, x_{2}, y_{2}, \psi_{2}, \varphi_{11}, \varphi_{12}, \varphi_{21}, \varphi_{22}\right]^{T}$ is chosen as the generalized coordinates of the system, the generalized forces $Q_{\varphi i j}=T_{\mathrm{e} i j}$ and the others are zero, in which $T_{\mathrm{e} i j}$ is the electromagnetic torque of motor $i j$ on vibrating machine $i$.

Substituting the coordinates of the corresponding points into Eqs (3), (9) and (10) and applying Lagranges' Eq. (11) to them lead to the differential equations of motion of the system. Usually, $m_{i j}<<m_{i}, \psi<<1$ and $\psi_{i}<<1$. Hence, the inertia coupling resulting from the asymmetry of the eccentric lump can be neglected during the running process of the system. Therefore, the differential equations of motion of the system can be simplified as follows:

$$
\begin{aligned}
& m_{R} \ddot{x}+\left(f_{x}+4 f_{x 0}\right) \dot{x}-f_{x 0} \sum_{i=1}^{2} \dot{x}_{i}+\left(k_{x}+4 k_{x 0}\right) x-k_{x 0} \sum_{i=1}^{2} x_{i}=0 \\
& m_{R} \ddot{y}+\left(f_{y}+4 f_{y 0}\right) \dot{x}-f_{y 0} \sum_{i=1}^{2} \dot{y}_{i}+\left(k_{y}+4 k_{y 0}\right) y-k_{y 0} \sum_{i=1}^{2} y_{i}=0 \\
& J_{R} \ddot{\psi}+f_{\psi} \dot{\psi}-\sum_{j=1}^{2} f_{\psi y j} \dot{y}_{j}-f_{y 0} l_{0}^{2} \sum_{j=1}^{2} \dot{\psi}_{j}+k_{\psi} \psi-\sum_{j=1}^{2} k_{\psi y j} y_{j}-k_{y 0} l_{0}^{2} \sum_{j=1}^{2} \psi_{j}=0 \\
& m_{i} \ddot{x}_{i}+f_{x 0} \dot{x}_{i}-f_{x 0} \dot{x}+k_{x 0} x_{i}-k_{x 0} x=\sum_{j=1}^{2}(-1)^{j-1} m_{i j} r_{i j}\left(\dot{\varphi}_{i j}^{2} \cos \varphi_{i j}+\ddot{\varphi}_{i j} \sin \varphi_{i j}\right)
\end{aligned}
$$




$$
\begin{aligned}
& m_{i} \ddot{y}_{i}+f_{y 0} \dot{y}_{i}-f_{y 0} \dot{y}-f_{y i \psi} \dot{\psi}+k_{y 0} y_{i}-k_{y 0} y-k_{y i \psi} \psi=\sum_{j=1}^{2} m_{i j} r_{i j}\left(\dot{\varphi}_{i j}^{2} \sin \varphi_{i j}-\ddot{\varphi}_{i j} \cos \varphi_{i j}\right) \\
& J_{0} \ddot{\psi}_{i}+f_{y 0} l_{0}^{2} \dot{\psi}_{i}-f_{y 0} l_{0}^{2} \dot{\psi}+k_{y 0} l_{0}^{2} \psi_{i}-k_{y 0} l_{0}^{2} \psi= \\
& \sum_{j=1}^{2}(-1)^{j-1} m_{i j} r_{i j} l_{a i}\left(\dot{\varphi}_{i j}^{2} \sin \left(\varphi_{i j}-\beta\right)-\ddot{\varphi}_{i j} \cos \left(\varphi_{i j}-\beta\right)\right) \\
& \left(J_{0 i 1}+m_{i 1} r_{i 1}^{2}\right) \ddot{\varphi}_{i 1}+f_{i 1} \dot{\varphi}_{i 1}=T_{\mathrm{e} i 1}-m_{i 1} r_{i 1}\left(\ddot{y}_{i} \cos \varphi_{i 1}-\ddot{x}_{i} \sin \varphi_{i 1}-l_{a i} \ddot{\psi}_{i} \cos \left(\varphi_{i 1}-\beta\right)\right) \\
& \left(J_{0 i 2}+m_{i 2} r_{i 2}^{2}\right) \ddot{\varphi}_{i 2}+f_{i 2} \dot{\varphi}_{i 2}=T_{\mathrm{e} i 2}-m_{i 2} r_{i 2}\left(\ddot{y}_{i} \cos \varphi_{i 2}+\ddot{x}_{i} \sin \varphi_{i 2}+l_{0} \ddot{\psi}_{i} \cos \left(\varphi_{i 2}-\beta\right)\right) \\
& i=1,2 .
\end{aligned}
$$

with

$$
\begin{aligned}
f_{\psi} & =f_{y} l_{a}^{2}+2 f_{y 0}\left(l_{1}^{2}+l_{2}^{2}+2 l_{0}^{2}\right), \quad f_{\psi y 1}=f_{y 0} l_{1}, \quad f_{\psi y 2}=f_{y 0} l_{2}, \quad f_{\psi y 3}=-f_{y 0} l_{2}, \quad f_{\psi y 4}=-f_{y 0} l_{1}, \\
k_{\psi} & =k_{y} l_{a}^{2}+2 k_{y 0}\left(l_{1}^{2}+l_{2}^{2}+2 l_{0}^{2}\right), \quad k_{\psi y 1}=k_{y 0} l_{1}, \quad k_{\psi y 2}=k_{y 0} l_{2}, \quad k_{\psi y 3}=-k_{y 0} l_{2}, \quad k_{\psi y 4}=-k_{y 0} l_{1} \\
f_{y 1 \psi} & =f_{y 0} l_{1}, \quad f_{y 2 \psi}=f_{y 0} l_{2}, \quad f_{y 3 \psi}=-f_{y 0} l_{2}, \quad f_{y 4 \psi}=-f_{y 0} l_{1}, \quad k_{y 1 \psi}=k_{y 0} l_{1}, \quad k_{y 2 \psi}=k_{y 0} l_{2}, \\
k_{y 2 \psi} & =-k_{y 0} l_{2}, \quad k_{y 4 \psi}=-k_{y 0} l_{1} .
\end{aligned}
$$

\section{Synchronization of the vibrating system}

When the system operates in the steady-state, the instantaneous average phase of the four unbalanced rotors is assumed to be $\varphi$, and their instantaneous average angular velocity is $\dot{\varphi}$. If the average value of $\dot{\varphi}$ is assumed to be $\omega_{m}$ and the coefficient of the instantaneous change of $\dot{\varphi}$ is assumed to be $\nu_{0}, \dot{\varphi}$ can be expressed as [18]

$$
\dot{\varphi}=\left(1+\nu_{0}\right) \omega_{m}
$$

Assuming

$$
\begin{gathered}
\alpha_{1}=\frac{1}{2}\left(\varphi_{11}-\varphi_{22}\right), \quad \alpha_{2}=\frac{1}{2}\left(\varphi_{21}-\varphi_{22}\right), \quad \alpha_{3}=\frac{1}{4}\left(\varphi_{11}+\varphi_{12}-\varphi_{21}-\varphi_{22}\right), \quad \dot{\alpha}_{i}=\nu_{i} \omega_{m}(i=1,2,3), \\
\dot{\varphi}_{11}=\left(1+\varepsilon_{1}\right) \omega_{m}, \quad \dot{\varphi}_{12}=\left(1+\varepsilon_{2}\right) \omega_{m}, \quad \dot{\varphi}_{21}=\left(1+\varepsilon_{3}\right) \omega_{m}, \quad \dot{\varphi}_{22}=\left(1+\varepsilon_{4}\right) \omega_{m},
\end{gathered}
$$

we have

$$
\begin{aligned}
& \varphi_{11}=\varphi+\alpha_{1}+\alpha_{3}, \quad \varphi_{12}=\varphi-\alpha_{1}+\alpha_{3}, \quad \varphi_{21}=\varphi+\alpha_{2}-\alpha_{3}, \quad \varphi_{21}=\varphi-\alpha_{2}-\alpha_{3} \\
& \dot{\varphi}_{11}=\left(1+\varepsilon_{1}\right) \omega_{m}, \varepsilon_{1}=\nu_{0}+\nu_{1}+\nu_{3}, \quad \dot{\varphi}_{12}=\left(1+\varepsilon_{2}\right) \omega_{m}, \varepsilon_{2}=\nu_{0}-\nu_{1}+\nu_{3}, \\
& \dot{\varphi}_{21}=\left(1+\varepsilon_{3}\right) \omega_{m}, \varepsilon_{3}=\nu_{0}+\nu_{2}-\nu_{3}, \quad \dot{\varphi}_{22}=\left(1+\varepsilon_{4}\right) \omega_{m}, \varepsilon_{4}=\nu_{0}-\nu_{2}-\nu_{3} .
\end{aligned}
$$

When the vibrating system operates in the steady-state, the slip of an induction motor is very small. Hence, the effect of the angular accelerations of the unbalance rotors on the response of the vibrating system can be neglected, i.e., $\ddot{\varphi}_{i j} \approx 0[18-20]$. On the other hand, the natural frequency of the vibrating system is far less than its operating one and its damping is very small [18-20]. In this case, the effect of the fluctuation of the angular velocities on the amplitude and phase angle of the response can also be neglected, i.e., the amplitude and phase angles of response of the vibrating system can be expressed by using $\omega_{m}$ and neglecting $\nu_{i}$. Therefore, the responses of the vibrating system can be expressed as follows:

$$
x=r_{\mathrm{ma}} r_{0} \mu_{x} \sum_{i=1}^{2}\left(\cos \left(\varphi_{i 1}-\gamma_{x \mathrm{e}}\right)-\cos \left(\varphi_{i 2}-\gamma_{\mathrm{e} x}\right)\right)
$$




$$
\begin{aligned}
& y=r_{\mathrm{ma}} r_{0} \mu_{y} \sum_{i=1}^{2} \sum_{j=1}^{2} \sin \left(\varphi_{i j}-\gamma_{\mathrm{e} y}\right) \\
& \psi=\frac{r_{\mathrm{ma}} r_{0} \mu_{\mathrm{e} \psi}}{l_{\mathrm{e}}}\left[\varsigma_{y 0} \sum_{i=1}^{2} r_{\mathrm{e} i} \sum_{j=1}^{2} \sin \left(\varphi_{i j}-\gamma_{\mathrm{e} \psi}\right)+\varsigma_{\psi 0} r_{\mathrm{ea}} \sum_{i=1}^{2}\left(\sin \left(\varphi_{i 1}-\beta-\gamma_{\mathrm{e} \psi}\right)-\sin \left(\varphi_{i 2}-\beta-\gamma_{\mathrm{e} \psi}\right)\right)\right] \\
& x_{i}=r_{m} r_{0} \mu_{x 0}\left[-\left(\cos \left(\varphi_{i 1}+\gamma_{x 0}\right)-\cos \left(\varphi_{i 2}+\gamma_{x 0}\right)+\varsigma_{x 0} \varsigma_{\mathrm{e} x} \sum_{j=1}^{2}\left(\cos \left(\varphi_{j l}-\gamma_{\mathrm{e} x 0}\right)-\cos \left(\varphi_{j l}-\gamma_{\mathrm{e} x 0}\right)\right)\right]\right. \\
& y_{i}=r_{m} r_{0} \mu_{y 0}\left[-\sum_{j=1}^{2} \sin \left(\varphi_{i j}+\gamma_{y 0}\right)+\sum_{j=1}^{2} \sum_{l=1}^{2} \varsigma_{y 0}\left(\varsigma_{\mathrm{e} y}+r_{\mathrm{e} i} r_{\mathrm{e} l} \varsigma_{\mathrm{e} \psi 0}\right) \sin \left(\varphi_{j l}-\gamma_{\mathrm{e} y 0}\right)+\right. \\
& r_{\mathrm{e} i} r_{\mathrm{ea}} \varsigma_{\mathrm{e} \psi 0} \varsigma_{\psi} \sum_{j=1}^{4}\left(\sin \left(\varphi_{j 1}-\beta-\gamma_{\mathrm{e} y \psi 0}\right)-\sin \left(\varphi_{j 2}-\beta-\gamma_{\mathrm{e} y \psi 0}\right)\right] \\
& \psi_{i}=\frac{r_{m} r_{0} \mu_{\psi 0}}{l_{\mathrm{e} 0}}\left[-r_{\mathrm{ea}} \sum_{j=1}^{2}\left(\sin \left(\varphi_{i 1}+\gamma_{\psi 0}\right)-\sin \left(\varphi_{i 2}+\gamma_{\psi 0}\right)\right)+\varsigma_{y 0} \varsigma_{\psi 0} \sum_{j=1}^{2} r_{\mathrm{e} 0 j} \sum_{l=1}^{2} \sin \left(\varphi_{j l}-\gamma_{\mathrm{e} \psi y 0}\right)+\right. \\
& \left.\varsigma_{\psi 0} \varsigma_{\mathrm{e} \psi} r_{\mathrm{ea}} \sum_{j=1}^{2}\left(\sin \left(\varphi_{j 1}-\gamma_{\mathrm{e} \psi 0}\right)-\sin \left(\varphi_{j 2}-\gamma_{\mathrm{e} \psi 0}\right)\right)\right]
\end{aligned}
$$

where $\gamma_{\mathrm{e} x}, \gamma_{\mathrm{e} y}, \gamma_{\mathrm{e} \psi}, \gamma_{\mathrm{e} x 0}, \gamma_{\mathrm{e} y 0}, \gamma_{\mathrm{e} \psi 0}, \gamma_{\mathrm{e} y \psi 0}, \pi-\gamma_{x 0}, \pi-\gamma_{y 0}$ and $\pi-\gamma_{\psi 0}$ are the phase angles.

$$
\begin{aligned}
& \varsigma_{x 0}=\frac{\eta_{x 0}^{2}}{\eta_{x 0}^{2}-1}, \quad k_{\mathrm{e} x}=k_{x}-4 \varsigma_{x 0} k_{x 0}, \quad \omega_{\mathrm{e} x}=\sqrt{\frac{k_{\mathrm{e} x}}{m_{R}}}, \quad \eta_{\mathrm{e} x}=\frac{\omega_{\mathrm{e} x}}{\omega_{m}}, \quad \mu_{x}=\frac{\varsigma_{x 0}}{\eta_{\mathrm{e} x}^{2}-1}, \\
& \mu_{x 0}=\frac{1}{\eta_{x 0}^{2}-1}, \quad l_{\mathrm{e} 0}=\sqrt{\frac{J_{0}}{m}}, \quad \varsigma_{\mathrm{e} x}=\frac{\eta_{\mathrm{e} x 0}^{2}}{\eta_{\mathrm{e} x}^{2}-1}, \quad \eta_{\mathrm{e} x 0}=\frac{\sqrt{k_{x 0} / m_{R}}}{\omega_{m}}, \quad \varsigma_{y 0}=\frac{\eta_{y 0}^{2}}{\eta_{y 0}^{2}-1}, \\
& k_{\mathrm{e} y}=k_{y}-4 \varsigma_{y 0} k_{y 0}, \quad \omega_{\mathrm{e} y}=\sqrt{\frac{k_{\mathrm{e} y}}{m_{R}}}, \quad \mu_{y}=\frac{\varsigma_{y 0}}{\eta_{\mathrm{e} y}^{2}-1}, \quad \mu_{y 0}=\frac{1}{\eta_{y 0}^{2}-1}, \quad \varsigma_{\mathrm{e} y}=\frac{\eta_{\mathrm{e} y 0}^{2}}{\eta_{\mathrm{e} y}^{2}-1}, \\
& \eta_{\mathrm{e} y 0}=\frac{\sqrt{k_{y 0} / m_{R}}}{\omega_{m}}, \quad \varsigma_{\psi 0}=\frac{\eta_{\psi 0}^{2}}{\eta_{\psi 0}^{2}-1}, \eta_{\psi 0}=\frac{\sqrt{k_{y 0} l_{0}^{2} / J_{0}}}{\omega_{m}}, \quad r_{\mathrm{e} 0}=\frac{l_{0}}{l_{\mathrm{e} 0}}, r_{\mathrm{ma}}=\frac{m_{0}}{m_{R}}, r_{\mathrm{m}}=\frac{m_{0}}{m}, \\
& m_{1}=m_{2}=m, m_{11}=m_{12}=m_{21}=m_{22}=m_{0} \text {, } \\
& k_{\mathrm{e} \psi}=k_{\psi}-2 k_{y 0 \varsigma_{y 0}}\left(l_{1}^{2}+l_{2}^{2}\right)-4 k_{y 0} l_{0}^{2} \varsigma_{\psi 0}, \quad r_{\mathrm{e} 1}=-r_{\mathrm{e} 2}=\frac{l_{1}}{l_{\mathrm{e}}}, r_{\mathrm{ea}}=\frac{l_{a}}{l_{\mathrm{e}}}, \quad l_{\mathrm{e}}=\sqrt{\frac{J_{R}}{m_{R}}}, \\
& \omega_{\mathrm{e} \psi}=\sqrt{\frac{k_{\mathrm{e} \psi}}{J_{R}}}, \quad \eta_{\mathrm{e} \psi}=\frac{\omega_{\mathrm{e} \psi}}{\omega_{m}}, \quad \varsigma_{y y 0}=\frac{\eta_{c y}^{2} \eta_{y 0}^{2}}{\left(\eta_{y}^{2}-1\right)\left(\eta_{y 0}^{2}-1\right)-4 \eta_{c y}^{2} \eta_{y 0}^{2}}, \quad \mu_{\mathrm{e} \psi}=\frac{1}{\eta_{\mathrm{e} \psi}^{2}-1}, \\
& \varsigma_{\mathrm{e} \psi}=\frac{\eta_{\mathrm{e} \psi 0}^{2}}{\eta_{\mathrm{e} \psi}^{2}-1}, \quad \eta_{\mathrm{e} \psi 0}=\frac{\sqrt{k_{y 0} l_{0}^{2} / J_{0}}}{\omega_{m}} .
\end{aligned}
$$

We differentiate $x_{i}, y_{i}$ and $\psi_{i}$ in Eq. (15) with respect to time $t$ by the chain rule (applied to each component of $\alpha_{1}$, $\alpha_{2}, \alpha_{3}$ and $\varphi$ ) to obtain $\ddot{x}_{i}, \ddot{y}_{i}$ and $\ddot{\psi}_{i}$, respectively. Then substituting $\ddot{x}_{i}, \ddot{y}_{i}$ and $\ddot{\psi}_{i}$ into the differential equations of motion for the four unbalanced rotors in Eq. (12) and integrating them over $\varphi=0 \sim 2 \pi$ and neglecting the high order terms of $\varepsilon_{i j}$, considering Eq. (14), we obtain the average differential equations of the four parameters as 
follows:

$$
\begin{gathered}
\left(J_{\mathrm{r} i}+m_{0} r_{0}^{2}\right) \omega_{m} \dot{\bar{\varepsilon}}_{i}+f_{\mathrm{d} i} \omega_{m}\left(1+\bar{\varepsilon}_{i}\right)=\bar{T}_{\mathrm{e} i}-m r^{2} \omega_{m}\left(\sum_{j=1}^{4} \chi_{i j}^{\prime} \dot{\bar{\varepsilon}}_{j}+\omega_{m} \sum_{j=1}^{4} \chi_{i j} \bar{\varepsilon}_{j}\right)-\chi_{\mathrm{f} i}-\chi_{\mathrm{a} i} \\
i=1,2,3,4 .
\end{gathered}
$$

where $J_{\mathrm{r} 1}=J_{011}, J_{\mathrm{r} 2}=J_{012}, J_{\mathrm{r} 3}=J_{021}, J_{\mathrm{r} 4}=J_{022}, f_{\mathrm{d} 1}=f_{11}, f_{\mathrm{d} 2}=f_{12}, f_{\mathrm{d} 3}=f_{21}, f_{\mathrm{d} 4}=f_{22}, \bar{T}_{\mathrm{e} 1}=\bar{T}_{\mathrm{e} 11}$, $\bar{T}_{\mathrm{e} 2}=\bar{T}_{\mathrm{e} 12}, \bar{T}_{\mathrm{e} 3}=\bar{T}_{\mathrm{e} 21}, \bar{T}_{\mathrm{e} 4}=\bar{T}_{\mathrm{e} 22}$.

Compared with the change of $\varphi\left(\dot{\varphi}=\omega_{\mathrm{m}}\right)$ with respect to time $t$, that of $\varepsilon_{i}$ and $\dot{\varepsilon}_{i}$ are very small, so $\varepsilon_{i}$ and $\dot{\varepsilon}_{i}$ are considered to be slow-changing parameters in this study. During the aforementioned integration, $\varepsilon_{i}, \dot{\varepsilon}_{i}$ and $\alpha_{i}$ are assumed to be the middle values of their integration $\bar{\varepsilon}_{i}, \dot{\bar{\varepsilon}}_{i}$ and $\bar{\alpha}_{i}$, respectively. The coefficients of $\bar{\varepsilon}_{i}, \dot{\bar{\varepsilon}}_{i}$ in Eq. (16) are listed in Appendix A. In engineering, the damping of the vibrating system is very small, hence the sine of phase angles in $\chi_{i j}^{\prime}$ and $\chi_{i j}$ are neglected [18-20].

When a asynchronous motor operates in the vicinity of the angular velocity of $\omega_{m}$, the electromagnetic torque can be expressed as the following [18]:

$$
\bar{T}_{\mathrm{e} i}=\bar{T}_{\mathrm{e} 0 i}-\bar{k}_{\mathrm{e} i} \bar{\varepsilon}_{i}
$$

where $T_{\mathrm{e} 0 i}$ and $k_{\mathrm{e} 0 i}$ are the electromagnetic torque and the stiffness coefficient of angular velocity of the motor when its angular velocity is $\omega_{m}$, respectively.

Because the moment of inertia of each motor's rotor is much smaller than that of the unbalanced rotor, it can be neglected in Eq. (16). Introducing the following non-dimensional parameters

$$
\begin{aligned}
\rho_{i} & =1-W_{\mathrm{c} 0} / 2, \quad i=1,2,3,4 ; \\
\kappa_{1} & =\frac{k_{\mathrm{e} 01}}{m_{0} r_{0}^{2} \omega_{m}^{2}}+\frac{f_{\mathrm{d} 1}}{m_{0} r_{0}^{2}}+W_{\mathrm{s} 1}, \quad \kappa_{2}=\frac{k_{\mathrm{e} 02}}{m_{0} r_{0}^{2} \omega_{m}^{2}}+\frac{f_{\mathrm{d} 2}}{m_{0} r_{0}^{2}}+W_{\mathrm{s} 2}, \\
\kappa_{3} & =\frac{k_{\mathrm{e} 03}}{m_{0} r_{0}^{2} \omega_{m}^{2}}+\frac{f_{\mathrm{d} 3}}{m_{0} r_{0}^{2}}+W_{\mathrm{s} 2}, \quad \kappa_{4}=\frac{k_{\mathrm{e} 04}}{m_{0} r_{0}^{2} \omega_{m}^{2}}+\frac{f_{\mathrm{d} 4}}{m_{0} r_{0}^{2}}+W_{\mathrm{s} 2} .
\end{aligned}
$$

into Eq. (16), dividing each formula by $m_{0} r_{0}^{2} \omega_{m}$, rearranging them and writing it in the matrix form, we obtain

$$
\boldsymbol{A} \dot{\bar{\varepsilon}}=\boldsymbol{B} \bar{\varepsilon}+\boldsymbol{u}
$$

where

$$
\begin{aligned}
& \boldsymbol{A}=\left[\begin{array}{llll}
\rho_{1} & \chi_{12}^{\prime} & \chi_{13}^{\prime} & \chi_{14}^{\prime} \\
\chi_{21}^{\prime} & \rho_{2} & \chi_{23}^{\prime} & \chi_{24}^{\prime} \\
\chi_{31}^{\prime} & \chi_{32}^{\prime} & \rho_{3} & \chi_{34}^{\prime} \\
\chi_{41}^{\prime} & \chi_{42}^{\prime} & \chi_{43}^{\prime} & \rho_{4}
\end{array}\right], \quad \boldsymbol{B}=\left[\begin{array}{llll}
\kappa_{1} & \chi_{12} & \chi_{13} & \chi_{14} \\
\chi_{21} & \kappa_{2} & \chi_{23} & \chi_{24} \\
\chi_{31} & \chi_{32} & \kappa_{3} & \chi_{34} \\
\chi_{41} & \chi_{42} & \chi_{43} & \kappa_{4}
\end{array}\right], \\
& \overline{\boldsymbol{\varepsilon}}=\left\{\bar{\varepsilon}_{1} \bar{\varepsilon}_{2} \bar{\varepsilon}_{3} \bar{\varepsilon}_{4}\right\}^{T}, \quad \boldsymbol{u}=\left\{u_{1} u_{2} u_{3} u_{4}\right\} . \\
& u_{1}=\frac{\bar{T}_{\mathrm{e} 01}}{m_{0} r_{0}^{2} \omega_{m}}-\frac{f_{\mathrm{d} 1}}{m_{0} r_{0}^{2}}-\frac{\chi_{\mathrm{f} 1}+\chi_{\mathrm{a} 1}}{m_{0} r_{0}^{2} \omega_{m}}, \quad u_{2}=\frac{\bar{T}_{\mathrm{e} 02}}{m_{0} r_{0}^{2} \omega_{m}}-\frac{f_{\mathrm{d} 2}}{m_{0} r_{0}^{2}}-\frac{\chi_{\mathrm{f} 2}+\chi_{\mathrm{a} 2}}{m_{0} r_{0}^{2} \omega_{m}}, \\
& u_{3}=\frac{\bar{T}_{\mathrm{e} 03}}{m_{0} r_{0}^{2} \omega_{m}}-\frac{f_{\mathrm{d} 3}}{m_{0} r_{0}^{2}}-\frac{\chi_{\mathrm{f} 3}+\chi_{\mathrm{a} 3}}{m_{0} r_{0}^{2} \omega_{m}}, \quad u_{2}=\frac{\bar{T}_{\mathrm{e} 04}}{m_{0} r_{0}^{2} \omega_{m}}-\frac{f_{\mathrm{d} 4}}{m_{0} r_{0}^{2}}-\frac{\chi_{\mathrm{f} 4}+\chi_{\mathrm{a} 4}}{m_{0} r_{0}^{2} \omega_{m}}
\end{aligned}
$$

If the trial solution of Eq. (18) exists and is stable, the four unbalanced rotors can implement self-synchronization. Equation (18) is the average differential equations of the angular velocity disturbances of the four unbalanced rotors over their average period and called the non-dimensional coupling equation of the four unbalanced rotors. The analytical approach used in this paper converts the problem of synchronization of the multiple unbalanced rotors in a vibrating system into that of existence and stability of trial solution for their non-dimensional coupling equation. 


\subsection{Conditions of implementing synchronization}

Inserting $\boldsymbol{u}=\mathbf{o}$ into Eq. (18) yields

$$
T_{\mathrm{o} i}=\chi_{\mathrm{f} i}+\chi_{\mathrm{a} i}, \quad i=1,2,3,4 .
$$

where $T_{\mathrm{o} i}$ is the differnece between the electromagnetic torque of the motor $i$ and the frictional torque of its rotor and called the output torque of the motor $i, T_{\mathrm{o} i}=\bar{T}_{\mathrm{e} 0 i}-f_{\mathrm{d} i} \omega_{m}$.

Subtracting the second formula in Eq. (19) from the first one yields

$$
\begin{aligned}
\Delta T_{\mathrm{o} 12} & =T_{o 1}-T_{o 2} \\
& =m_{0} r_{0}^{2} \omega_{\mathrm{m}}^{2}\left[\left(W_{\mathrm{c} 0}-W_{y}-W_{\psi}\right) \sin 2 \bar{\alpha}_{1}+2\left(W_{\psi}-W_{y}\right) \cos 2 \bar{\alpha}_{3} \cos \bar{\alpha}_{2} \sin \bar{\alpha}_{1}\right]
\end{aligned}
$$

where $W_{y}=r_{m} \mu_{y 0} \varsigma_{y 0} \varsigma_{\mathrm{e} y} \cos \gamma_{\mathrm{e} y 0}$ and $W_{\psi}=r_{m} \mu_{\psi 0} \varsigma_{y 0} \varsigma_{\mathrm{e} \psi 0} r_{\mathrm{e} 1}^{2} \cos \gamma_{e \psi y 0}$.

Subtracting the fourth formula in Eq. (19) from the third one yields

$$
\begin{aligned}
\Delta T_{\mathrm{o} 34} & =T_{o 3}-T_{o 4} \\
& =m_{0} r_{0}^{2} \omega_{\mathrm{m}}^{2}\left[\left(W_{\mathrm{c} 0}-W_{y}-W_{\psi}\right) \sin 2 \bar{\alpha}_{2}+2\left(W_{\psi}-W_{y}\right) \cos 2 \bar{\alpha}_{3} \cos \bar{\alpha}_{1} \sin \bar{\alpha}_{2}\right]
\end{aligned}
$$

Subtracting the sum of the last two formulae in Eq. (19) from that of the first two ones yields

$$
\begin{aligned}
\Delta T_{o} & =T_{o 1}+T_{o 2}-T_{o 3}-T_{o 4} \\
& =4 m_{0} r_{0}^{2} \omega_{m}^{2}\left(W_{\psi}-W_{y}\right) \cos \bar{\alpha}_{1} \cos \bar{\alpha}_{2} \sin 2 \bar{\alpha}_{3}
\end{aligned}
$$

In order to obtain the greater torque of frequency capture and the stable magnitude of a vibrating frame, a vibrating machine is always designed to be the over resonant type, i.e., its frequency of operation is greater than its natural one. Usually, the frequency of operation is in the range of 4 to 5 times its natural one [14]. In this case, the amplitudes of response of the vibrating system are almost independent of the exciting frequency and constants [14]. Assuming

$$
\omega_{m} \geqslant 4 \omega_{n}
$$

where $\omega_{n}$ represents the natural frequency of the vibrating systems, then we have

$$
\varsigma_{x 0} \geqslant \frac{1}{15}, \quad \varsigma_{y 0} \geqslant \frac{1}{15}, \quad \varsigma_{\psi 0} \geqslant \frac{1}{15}
$$

Hence, in order to increase the torques of frequency capture between two of the four unbalanced rotors that are not installed on the same vibrating rigid frame, the natural frequency of the isolation frame is designed to be much higher than the operation frequency of the vibrating system. Assuming

$$
\omega_{\mathrm{e} x} \geqslant 4 \omega_{m}, \quad \omega_{\mathrm{e} y} \geqslant 4 \omega_{m}, \quad \omega_{\mathrm{e} \psi} \geqslant 4 \omega_{m}
$$

we have

$$
\varsigma_{\mathrm{e} x} \geqslant \frac{16}{15} \approx 1, \quad \varsigma_{\mathrm{e} y} \geqslant \frac{16}{15} \approx 1, \quad \varsigma_{\mathrm{e} \psi} \geqslant \frac{16}{15} \approx 1
$$

As shown in Eqs (24) and (26), it can be seen that $W_{\mathrm{c} 0}$ is much greater than $W_{y}$ and $W_{\psi}$. Hence, Equations (20) and (22) can be simplified as follows:

$$
\begin{aligned}
& \Delta T_{\mathrm{o} 12}=T_{\mathrm{C} 1} \sin 2 \bar{\alpha}_{1} \\
& \Delta T_{\mathrm{o} 34}=T_{\mathrm{C} 1} \sin 2 \bar{\alpha}_{2}
\end{aligned}
$$

where $T_{\mathrm{C} 1}=m_{0} r_{0}^{2} \omega_{\mathrm{m}}^{2} W_{\mathrm{c} 0}$ is called the first torque of frequency capture.

Then the phase differences between each pair of the unbalanced rotors on the same rigid frame can be expressed as

$$
\begin{aligned}
& 2 \bar{\alpha}_{1}=\arcsin \frac{\Delta T_{\mathrm{o} 12}}{T_{\mathrm{C} 1}} \\
& 2 \bar{\alpha}_{2}=\arcsin \frac{\Delta T_{\mathrm{o} 34}}{T_{\mathrm{C} 1}}
\end{aligned}
$$


As shown in Eq. (80), when the parameters of the vibrating system satisfy

$$
\left|T_{\mathrm{C} 1}\right| \geqslant \max \left\{\left|\Delta T_{\mathrm{o} 12}\right|,\left|\Delta T_{\mathrm{o} 34}\right|\right\}
$$

the solutions of $2 \bar{\alpha}_{1}$ and $2 \bar{\alpha}_{2}$ exist.

Equation (29) demonstrates that the condition that each pair of the unbalanced rotors on the same rigid frame is that the first torque of frequency capture is equal to or greater than the difference in the output torque between each pair of motors on the same rigid frame. When the two unbalanced rotors in a vibrating system with small damping rotate in opposite directions, the first torque of frequency capture is always much greater than the absolute value of the difference of output torque for each pair of the motors. Hence, $2 \bar{\alpha}_{1}$ and $2 \bar{\alpha}_{2}$ are close to 0 or $\pi[14,15]$. Therefore, $2 \bar{\alpha}_{3}$ can be approximately expressed as

$$
2 \bar{\alpha}_{3}=\arcsin \frac{\Delta T_{o}}{T_{\mathrm{C} 2}}
$$

where $T_{\mathrm{C} 2}$ is called the second torque of frequency capture for the considered vibrating system, $T_{\mathrm{C} 2}=$ $4 m_{0} r_{0}^{2} \omega_{m}^{2}\left(W_{\psi}-W_{y}\right)$.

Equation (30) demonstrates that the condition that the two vibrating machines can implement synchronization is that the second torque of frequency capture is equal to or greater than the absolute value of the output torque difference between the two pairs of the motors. i.e.,

$$
\left|T_{\mathrm{C} 2}\right| \geqslant\left|\Delta T_{o}\right|
$$

When the parameters of the vibrating system satisfy Eqs (29) and (31), the solutions of Eq. (19), denoted by $\bar{\alpha}_{10}, \bar{\alpha}_{20}$, $\bar{\alpha}_{30}$ and $\omega_{\mathrm{m} 0}$, can be determined by a numeric method.

\subsection{Condition of the synchronization stability}

When $\boldsymbol{u}=\mathbf{o}$, Eq. (18) is a generalized system [22]

$$
A_{0} \dot{\varepsilon}=B_{0} \varepsilon
$$

where $\boldsymbol{A}_{0}$ and $\boldsymbol{B}_{0}$ denote the values of $\boldsymbol{A}$ and $\boldsymbol{B}$ for $\bar{\alpha}_{1}=\bar{\alpha}_{10}, \bar{\alpha}_{2}=\bar{\alpha}_{20}, \bar{\alpha}_{3}=\bar{\alpha}_{30}$ and $\omega_{m}=\omega_{\mathrm{m} 0}$.

As shown in the expressions of $\chi_{i j}^{\prime}$ and $\chi_{i j}(i=1,2,3,4 ; j=1,2,3,4)$ in Appendix A, when the parameters of the vibrating system satisfies the follow condition:

$$
a_{i j}>0, \quad \operatorname{det}\left(\boldsymbol{A}_{2}\right)>0, \quad \operatorname{det}\left(\boldsymbol{A}_{3}\right)>0, \quad \operatorname{det}\left(\boldsymbol{A}_{0}\right)>0,
$$

the matrices $\boldsymbol{A}_{0}$ and $\boldsymbol{B}_{0}$ satisfy the generalized Lyapunov equations [22]:

$$
\begin{aligned}
& \boldsymbol{I}^{T} \boldsymbol{B}_{0}+\boldsymbol{B}_{0}^{T} \boldsymbol{I}=-2 \omega_{m} \operatorname{diag}\left\{\kappa_{1}, \kappa_{2}, \kappa_{3}, \kappa_{4}\right\} \\
& \boldsymbol{A}_{0}^{T} \boldsymbol{I}=\boldsymbol{I} \boldsymbol{A}_{0}>0
\end{aligned}
$$

where $\boldsymbol{I}$ is the unit matrix.

As shown in Appendix, when $\bar{\alpha}_{10}, \bar{\alpha}_{20}$ and $\bar{\alpha}_{30}$ satisfy

$$
\begin{aligned}
& -\pi / 2<2 \bar{\alpha}_{10}<\pi / 2, \quad-\pi / 2<2 \bar{\alpha}_{20}<\pi / 2, \quad-\pi / 2<2 \bar{\alpha}_{30}<\pi / 2, \\
& \pi / 2<2 \bar{\alpha}_{30}-\bar{\alpha}_{10}-\bar{\alpha}_{20}<\pi / 2, \quad \pi / 2<2 \bar{\alpha}_{30}+\bar{\alpha}_{10}+\bar{\alpha}_{20}<\pi / 2, \\
& \pi / 2<2 \bar{\alpha}_{30}-\bar{\alpha}_{10}+\bar{\alpha}_{20}<\pi / 2, \quad \pi / 2<2 \bar{\alpha}_{30}+\bar{\alpha}_{10}-\bar{\alpha}_{20}<\pi / 2 .
\end{aligned}
$$

Equations (33), (34) and (35) can be satisfied.

Therefore, the generalized system (Eq. (32)) is concessional and without pulse. If $\underset{\lim t \rightarrow \infty}{\boldsymbol{A} \varepsilon}=\mathbf{o}$, Eq. (32) is stable [22]. $\underset{\lim t \rightarrow \infty}{\varepsilon}=\mathbf{o}$ means that the electromagnetic torques of the four motors are stably balanced with the load torques that the vibrating system acts on them.

Linearizing Eq. (19) around $\bar{\alpha}_{10}, \bar{\alpha}_{20}, \bar{\alpha}_{30}$ and $\omega_{\mathrm{m} 0}$, and neglecting $W_{\mathrm{sc}}$ and $W_{\mathrm{sc} 0}$, as well as $f_{\mathrm{d} 1}, f_{\mathrm{d} 2}, f_{\mathrm{d} 3}$ and $f_{\mathrm{d} 4}$, we obtain 


$$
\begin{aligned}
& k_{\mathrm{e} 01}\left(\nu_{0}+\nu_{1}+\nu_{2}\right)=-\sum_{i=1}^{3}\left(\frac{\partial \chi_{\mathrm{a} 1}}{\partial \alpha_{i}}\right)_{0} \Delta \alpha_{i} \\
& k_{\mathrm{e} 02}\left(\nu_{0}+\nu_{1}-\nu_{2}\right)=-\sum_{i=1}^{3}\left(\frac{\partial \chi_{\mathrm{a} 2}}{\partial \alpha_{i}}\right)_{0} \Delta \alpha_{i}, \\
& k_{\mathrm{e} 03}\left(\nu_{0}-\nu_{1}+\nu_{3}\right)=-\sum_{i=1}^{3}\left(\frac{\partial \chi_{\mathrm{a} 3}}{\partial \alpha_{i}}\right)_{0} \Delta \alpha_{i}, \\
& k_{\mathrm{e} 04}\left(\nu_{0}-\nu_{1}-\nu_{3}\right)=-\sum_{i=1}^{3}\left(\frac{\partial \chi_{\mathrm{a} 4}}{\partial \alpha_{i}}\right)_{0} \Delta \alpha_{i},
\end{aligned}
$$

where $(\bullet)_{0}$ denotes the values for $\bar{\alpha}_{1}=\bar{\alpha}_{10}, \bar{\alpha}_{2}=\bar{\alpha}_{20}$ and $\bar{\alpha}_{3}=\bar{\alpha}_{30}$; and $\Delta \alpha_{i}=\bar{\alpha}_{i}-\bar{\alpha}_{i 0}, i=1,2,3$.

Summing Eqs (37)-(40), and rearranging them, we obtain

$$
v_{0}=\delta_{1} \nu_{1}+\delta_{2} \nu_{2}+\delta_{3} \nu_{3}
$$

where

$$
\begin{aligned}
& \delta_{1}=-\frac{k_{\mathrm{e} 01}+k_{\mathrm{e} 02}-k_{\mathrm{e} 03}-k_{\mathrm{e} 02}}{k_{\mathrm{e} 01}+k_{\mathrm{e} 02}+k_{\mathrm{e} 03}+k_{\mathrm{e} 02}}, \\
& \delta_{2}=-\frac{k_{\mathrm{e} 01}-k_{\mathrm{e} 02}}{k_{\mathrm{e} 01}+k_{\mathrm{e} 02}+k_{\mathrm{e} 03}+k_{\mathrm{e} 04}}, \\
& \delta_{3}=-\frac{k_{\mathrm{e} 03}-k_{\mathrm{e} 04}}{k_{\mathrm{e} 01}+k_{\mathrm{e} 02}+k_{\mathrm{e} 03}+k_{\mathrm{e} 04}} .
\end{aligned}
$$

It should be noticed that $\Delta \dot{\boldsymbol{\alpha}}=\left\{\Delta \dot{\alpha}_{1} \Delta \dot{\alpha}_{2} \Delta \dot{\alpha}_{2}\right\}^{T}=\left\{\nu_{1} \nu_{2} \nu_{3}\right\}^{T}$. Substituting Eq. (41) into Eqs (37)-(40), and writing them into the generalized system of $\Delta \boldsymbol{\alpha}=\left\{\Delta \alpha_{1} \Delta \alpha_{2} \Delta \alpha_{2}\right\}^{T}$ in the following manner: subtracting Eq. (38) from Eq. (37) as the first row, subtracting Eq. (39) from Eq. (40) as the second row, subtracting the sum of Eqs (39) and (40) from that of Eqs (37) and (38) as the third row, we obtain

$$
E \Delta \dot{\alpha}=D \Delta \alpha,
$$

where $\boldsymbol{E}=\left[e_{i j}\right]_{3 \times 3}$, and $\boldsymbol{D}=\left[d_{i j}\right]_{3 \times 3}$.

Equation (42) can be rewritten as:

$$
\Delta \dot{\boldsymbol{\alpha}}=\boldsymbol{C} \Delta \boldsymbol{\alpha}, \boldsymbol{C}=\boldsymbol{E}^{-1} \boldsymbol{D} .
$$

Herein, Eq. (43) is called the generalized system for the disturbance parameters of phase differences. Exponential time-dependence of the form $\Delta \boldsymbol{\alpha}=\boldsymbol{v} \exp (\lambda t)$ is now assumed, and inserted into Eq. (43), then solving the determinant equation $\operatorname{det}(\boldsymbol{C}-\lambda \boldsymbol{I})=0$, we obtain the characteristic equation for the eigenvalue $\lambda$ as the following:

$$
\lambda^{3}+c_{1} \lambda^{2}+c_{2} \lambda+c_{3}=0
$$

The zero solutions of Eq. (43) are stable only if all the roots of $\lambda$ in Eq. (44) have negative real parts. Using the Routh-Hurwitz criterion, Equation (45) satisfies the above requirements [11]:

$$
c_{1}>0, \quad c_{3}>0 \text { and } c_{1} c_{2}>c_{3} .
$$

$\underset{\lim t \rightarrow+\infty}{\Delta \boldsymbol{\alpha}}=\mathbf{0}$ means $\underset{\lim t \rightarrow+\infty}{\nu_{i}}=0, i=0,1,2,3$. Using Eq. (14), we have $\underset{\lim t \rightarrow+\infty}{\boldsymbol{\varepsilon}}=\mathbf{0}$, i.e., $\underset{\lim t \rightarrow+\infty}{\boldsymbol{A} \varepsilon}=\mathbf{0}$.

In engineering, the parameters of the four induction motors are usually chosen to be similar $[14,15]$, i.e.,

$$
\begin{aligned}
& k_{\mathrm{e} 11} \approx k_{\mathrm{e} 12} \approx k_{\mathrm{e} 21} \approx k_{\mathrm{e} 22} \approx k_{\mathrm{e} 0}, \\
& T_{\mathrm{e} 11} \approx T_{\mathrm{e} 12} \approx T_{\mathrm{e} 21} \approx T_{\mathrm{e} 22} .
\end{aligned}
$$


In this case, the matrix $\boldsymbol{E}$ is approximately expressed as

$$
\boldsymbol{E}=\operatorname{diag}\left\{4 k_{\mathrm{e} 0}, 2 k_{\mathrm{e} 0}, 2 k_{\mathrm{e} 0}\right\}
$$

From Eqs (28) and (30), we have $\sin \bar{\alpha}_{10} \approx 0, \sin \bar{\alpha}_{20} \approx 0$ and $\sin \bar{\alpha}_{30} \approx 0$. Then the matrices $\boldsymbol{D}$ and $\boldsymbol{E}$ can be also approximately simplified as two diagonal matrices. Hence, Eq. (43) can be simplified as follows:

$$
\begin{aligned}
& \Delta \dot{\alpha}_{1}=-m_{0} r_{0}^{2} \omega_{\mathrm{m} 0} W_{\mathrm{c} 0} \cos 2 \bar{\alpha}_{10} \Delta \alpha_{1} \\
& \Delta \dot{\alpha}_{2}=-m_{0} r_{0}^{2} \omega_{\mathrm{m} 0} W_{\mathrm{c} 0} \cos 2 \bar{\alpha}_{20} \Delta \alpha_{2} \\
& \Delta \dot{\alpha}_{3}=-4 m_{0} r_{0}^{2} \omega_{m}^{2}\left(W_{\psi}-W_{y}\right) \cos \bar{\alpha}_{10} \cos \bar{\alpha}_{20} \cos 2 \bar{\alpha}_{30} \Delta \alpha_{3}
\end{aligned}
$$

In a vibrating system with dual-motor drivers rotating in opposite directions, $W_{\mathrm{c} 0}$ is always greater than 0 , hence $2 \bar{\alpha}_{10}$ and $2 \bar{\alpha}_{20}$ are stabilized in the vicinity of $0[14,15,18]$. If $W_{\psi}>W_{y}, 2 \bar{\alpha}_{30}$ is stabilized in the vicinity of 0 ; if $W_{\psi}<W_{y}, 2 \bar{\alpha}_{30}$ is in the vicinity of $\pi$.

\section{Computer simulations}

In this section, computer simulations are carried out to verify the above theoretic results. The numeric algorithm is developed from that of the vibrating system with two unbalanced rotors [19]. The parameters of the present system are listed in Appendix B.

Figures 3 and 4 show the results of computer simulations for the parameter $l_{1}=0.8\left(W_{\psi}<W_{y}\right)$ and 2.5 $\left(W_{\psi}>W_{y}\right)$, respectively. Because the damping coefficient of the rotor 11 is smaller than that of the rotor 21 and the parameters for the two pairs of motors are the same, the difference in the rotational speed between the two unbalanced rotors on V1 is greater than that on V2 during the starting process of the system, as shown in Fig. 3d. Hence, the phase difference between the two unbalanced rotors on V1 is greater than that between the two unbalanced rotors on V2, as shown in Figs 3a and b. But with the increase of the rotational speeds of the four motors, the vibrations of the two vibrating frames are excited and the first torque of frequency capture plays the role of synchronizing each pair of the unbalanced rotors. Then the phase difference between each pair of unbalanced rotors is stabilized in the vicinity of $0,2 \bar{\alpha}_{10}=0.04$ and $2 \bar{\alpha}_{20}=0.03$, as illustrated in Figs $3 \mathrm{a}$ and $\mathrm{b}$. At the same time, the second torque of frequency capture plays the role of synchronizing the rotational speeds of the unbalanced rotors on the two vibrating frames. Finally, the phase difference $2 \bar{\alpha}_{30}$ is stabilized in the vicinity of $2 \bar{\alpha}_{30}\left(2 \bar{\alpha}_{30}=3.19\right)$. When the rotational speed of each pair of unbalanced rotors passes through the natural frequency of each vibrating frame, the resonant responses are excited. But when the system operates in the steady-state, the vibrations of each vibrating frame in $x$ - and $\psi$-directions are rapidly attenuated due to the damping of the system and each vibrating frame undergoes an only vibration in $y$-direction, as shown in Figs 3e, $\mathrm{f}$ and $\mathrm{g}$ (vibrations of V2 are not shown in Fig. 3). Because the phase difference between the two pairs of unbalanced rotors is in the vicinity of $\pi$ and phase difference between each pair of unbalanced rotors is in the vicinity of 0 when the system operates in the steady-state, the exciting forces acting on the isolation in $x$ - and $y$-directions are cancelled mutually and the exciting torques in $\psi$-direction are superposed. Therefore, when the system operates in the steady-state, the isolation frame undergoes an only vibration in $\psi$-direction, as shown Fig. $3 \mathrm{~h}, \mathrm{i}$ and $\mathrm{j}$. For $l_{1}=2.5$, the phase difference between the two pairs of unbalanced rotors is in the vicinity of $0\left(2 \bar{\alpha}_{30}=0.25 \mathrm{rad}\right)$ and the isolation frame undergoes a only vibration in $y$-direction when the system operates in the steady-state, as shown in Fig. 4. These facts are consistent with the above theoretical results in Section 3.

\section{Conclusions}

This paper investigates synchronization of two self-synchronous vibrating machines on an isolation rigid frame. The mathematical model is set up by using Lagrange's equations. Using the modified average method of small parameters, we deduce the non-dimensional differential equation of the disturbance parameters for the angular velocities of the four unbalanced rotors, which includes the inertia coupling of the unbalanced rotors, the stiffness 

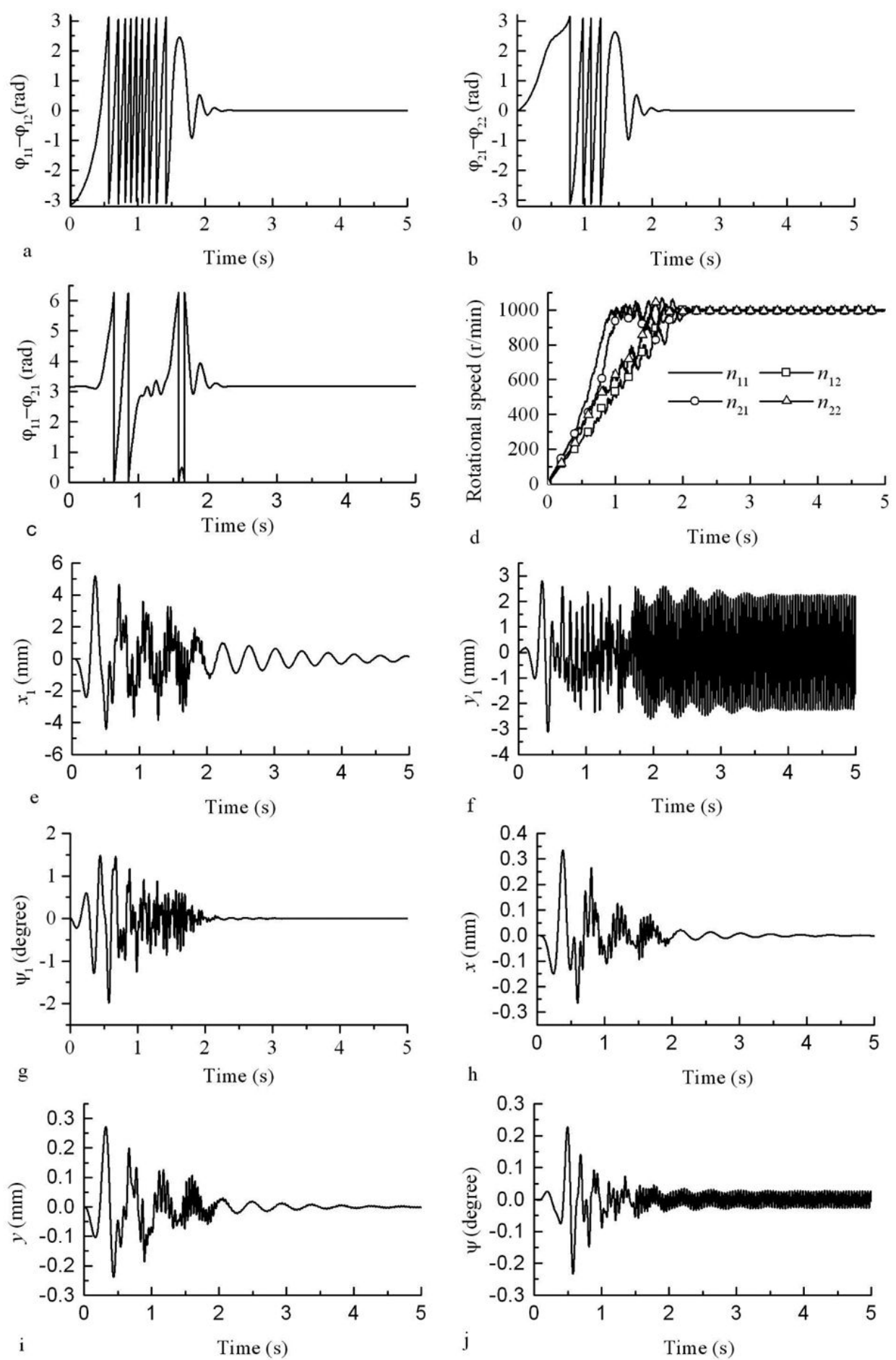

Fig. 3. Results of computer simulation for $l_{1}=0.8 \mathrm{~m}$ : a-phase difference between the two unbalanced rotors on V1; b-phase difference between the two unbalanced rotors on V2; c-phase difference between the unbalanced rotors 11 and 21; d-rotational velocities of the four unbalanced rotors; e-displacement of V1 in $x_{1}$-direction, f-displacement of V1 in $y_{1}$-direction; g-angular displacement of V1 in $\psi_{1}$-direction; h-displacement of the isolation frame in $x$-direction; i-displacement of the isolation frame in $y$-direction; $\mathrm{j}$-angular displacement of the isolation frame in $\psi$-direction. 

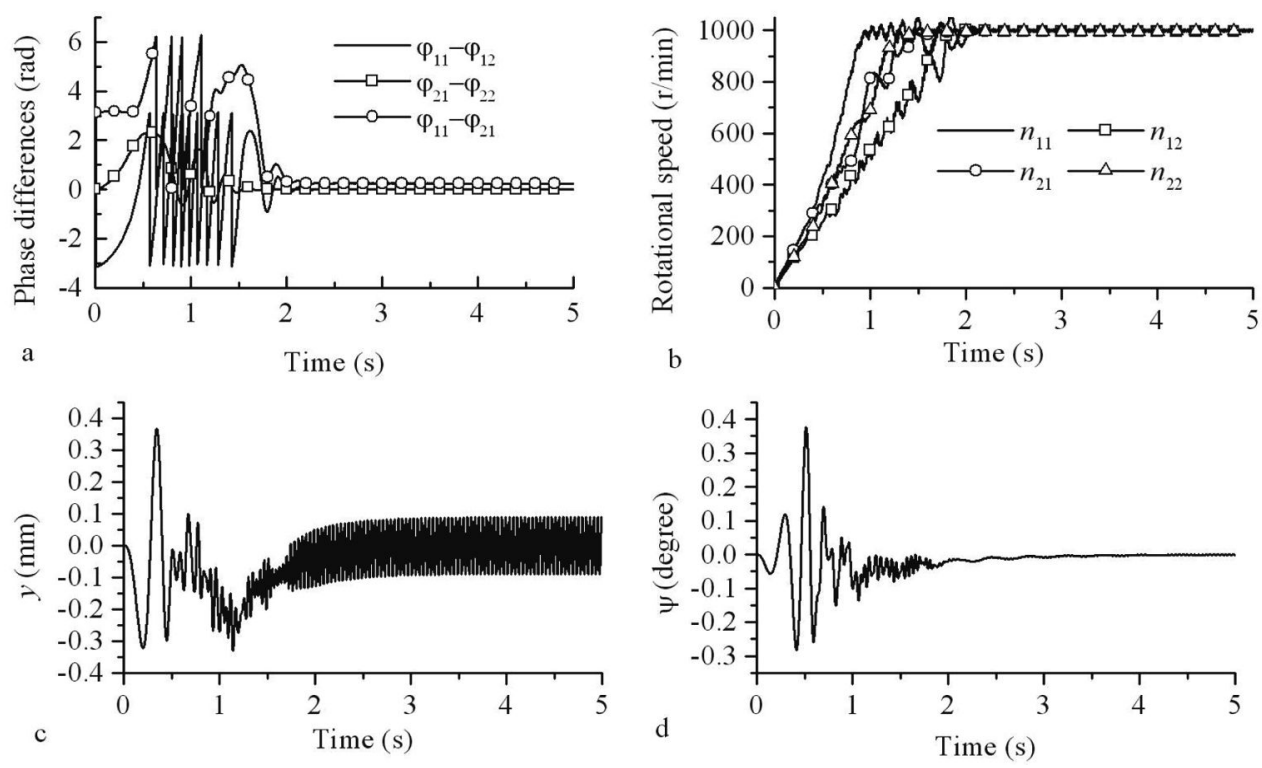

Fig. 4. Results of computer simulation for $l_{1}=2.5 \mathrm{~m}$ : a-phase differences; b-rotational speeds of the four unbalanced rotors; c-displacement of the isolation in $y$-direction; c-displacement of the isolation frame in $\psi$-direction.

coupling of angular velocity of the four motors, and the loading coupling of the four motors. This analytical approach converts the problem of synchronization for the four unbalanced rotors into the stability problem of a generalized system and a system of three first order differential equations for the three phase differences. In a vibrating system with small damping, the inertia coupling matrix of the four unbalanced rotors is symmetric, the stiffness coupling matrix is antisymmetrical and its diagonal elements are all negative. These facts make the generalized system satisfy the generalized Lyapunov equations when the inertia coupling matrix is positive definite. The condition of implementing synchronization is that the torque of frequency capture between each pair of the unbalanced rotors on a vibrating machine is greater than the absolute values of the output electromagnetic torque difference between each pair of motors, and that the torque of frequency capture between the two vibrating machines is greater than the absolute value of the output electromagnetic torque difference between the two pairs of motors on the two vibrating machines. The stability condition of synchronization of the two vibrating machines is that the inertia coupling matrix of the generalized system is definite positive, and that all the eigenvalues for the generalized system of three phase disturbance parameters have negative real parts.

\section{Acknowledgement}

This research is support by the National Science Foundation of China (Grant No: 51075063) and Program for Changjiang Scholars and Innovative Research Team in University.

\section{Appendix A Non-diagonal elements of matrices A and B}

$$
\begin{aligned}
& \chi_{\mathrm{f} 1}=\frac{1}{2} \omega_{m}\left[W_{s}+W_{\mathrm{sc} 12} \cos 2 \bar{\alpha}_{1}+W_{\mathrm{sc} 13} \cos \left(2 \bar{\alpha}_{3}+\bar{\alpha}_{1}-\bar{\alpha}_{2}\right)+W_{\mathrm{sc} 14} \cos \left(2 \bar{\alpha}_{3}+\bar{\alpha}_{1}+\bar{\alpha}_{2}\right)\right] \\
& \chi_{\mathrm{f} 2}=\frac{1}{2} \omega_{m}\left[W_{\mathrm{cs} 12} \cos 2 \bar{\alpha}_{1}+W_{\mathrm{s} 0}+W_{\mathrm{cs} 23} \cos \left(2 \bar{\alpha}_{3}-\bar{\alpha}_{1}-\bar{\alpha}_{2}\right)+W_{\mathrm{cs} 24} \cos \left(2 \bar{\alpha}_{3}-\bar{\alpha}_{1}+\bar{\alpha}_{2}\right)\right]
\end{aligned}
$$




$$
\begin{aligned}
& \chi_{\mathrm{f} 3}=\frac{1}{2} \omega_{m}\left[W_{\mathrm{cs} 13} \cos \left(2 \bar{\alpha}_{3}+\bar{\alpha}_{1}-\bar{\alpha}_{2}\right)+W_{\mathrm{cs} 23} \cos \left(2 \bar{\alpha}_{3}-\bar{\alpha}_{1}+\bar{\alpha}_{2}\right) W_{\mathrm{s} 0}+W_{\mathrm{cs} 34} \cos 2 \bar{\alpha}_{2}\right] \\
& \chi_{\mathrm{f} 4}=\frac{1}{2} \omega_{m}\left[W_{\mathrm{cs} 14} \cos \left(2 \bar{\alpha}_{3}+\bar{\alpha}_{1}+\bar{\alpha}_{2}\right)+W_{\mathrm{cs} 24} \cos \left(2 \bar{\alpha}_{3}-\bar{\alpha}_{1}+\bar{\alpha}_{2}\right)+W_{\mathrm{cs} 0} \cos 2 \bar{\alpha}_{2}+W_{\mathrm{s} 0}\right] \\
& \chi_{\mathrm{a} 1}=\frac{1}{2} \omega_{m}\left[W_{\mathrm{cc} 12} \sin 2 \bar{\alpha}_{1}+W_{\mathrm{cc} 13} \sin \left(2 \bar{\alpha}_{3}+\bar{\alpha}_{1}-\bar{\alpha}_{2}\right)+W_{\mathrm{cc} 24} \sin \left(2 \bar{\alpha}_{3}+\bar{\alpha}_{1}+\bar{\alpha}_{2}\right)\right] \\
& \chi_{\mathrm{a} 2}=\frac{1}{2} \omega_{m}\left[-W_{\mathrm{cc} 12} \sin 2 \alpha_{1}+W_{\mathrm{cc} 23} \sin \left(2 \bar{\alpha}_{3}-\bar{\alpha}_{1}-\bar{\alpha}_{2}\right)+W_{\mathrm{cc} 24} \sin \left(2 \bar{\alpha}_{3}-\bar{\alpha}_{1}+\bar{\alpha}_{2}\right)\right] \\
& \chi_{\mathrm{a} 3}=\frac{1}{2} \omega_{m}\left[-W_{\mathrm{cc} 13} \sin \left(2 \bar{\alpha}_{3}+\bar{\alpha}_{1}-\bar{\alpha}_{2}\right)-W_{\mathrm{cc} 23} \sin \left(2 \bar{\alpha}_{3}-\bar{\alpha}_{1}-\bar{\alpha}_{2}\right)+W_{\mathrm{cc} 0} \cos 2 \bar{\alpha}_{2}\right] \\
& \chi_{\mathrm{a} 4}=-\frac{1}{2} \omega_{m}\left[W_{\mathrm{cc} 14} \sin \left(2 \bar{\alpha}_{3}+\bar{\alpha}_{1}+\vec{\alpha}_{2}\right)+W_{\mathrm{cc} 24} \cos \left(2 \bar{\alpha}_{3}-\bar{\alpha}_{1}+\bar{\alpha}_{2}\right)+W_{\mathrm{cc} 0} \cos 2 \bar{\alpha}_{2}\right] \\
& \chi_{11}^{\prime}=-\frac{1}{2} W_{\mathrm{c} 0} \\
& \chi_{12}^{\prime}=\frac{1}{2} W_{\mathrm{cc} 12} \cos 2 \bar{\alpha}_{1} \\
& \left.\chi_{13}^{\prime}=\frac{1}{2} W_{\mathrm{cc} 13} \cos \left(2 \bar{\alpha}_{3}+\bar{\alpha}_{1}-\bar{\alpha}_{2}\right)\right) \\
& \left.\chi_{14}^{\prime}=\frac{1}{2} W_{\mathrm{cc} 14} \cos \left(2 \bar{\alpha}_{3}+\bar{\alpha}_{1}+\bar{\alpha}_{2}\right)\right) \\
& \chi_{21}^{\prime}=\frac{1}{2} W_{\mathrm{cc} 12} \cos 2 \bar{\alpha}_{1} \\
& \chi_{22}^{\prime}=-\frac{1}{2} W_{\mathrm{c} 0} \\
& \chi_{23}^{\prime}=\frac{1}{2} W_{\mathrm{cc}} \cos \left(2 \bar{\alpha}_{3}-\bar{\alpha}_{1}-\bar{\alpha}_{2}\right) \\
& \chi_{24}^{\prime}=\frac{1}{2} W_{\mathrm{cc}} \cos \left(2 \bar{\alpha}_{3}-\bar{\alpha}_{1}+\bar{\alpha}_{2}\right) \\
& \chi_{31}^{\prime}=\frac{1}{2} W_{\mathrm{cc} 13} \cos \left(2 \bar{\alpha}_{3}+\bar{\alpha}_{1}-\bar{\alpha}_{2}\right) \\
& \chi_{32}^{\prime}=\frac{1}{2} r_{\mathrm{m} 12} W_{\mathrm{cc} 23} \cos \left(2 \bar{\alpha}_{3}-\bar{\alpha}_{1}-\bar{\alpha}_{2}\right)
\end{aligned}
$$


$\chi_{33}^{\prime}=-\frac{1}{2} W_{\mathrm{c} 0}$

$\chi_{34}^{\prime}=\frac{1}{2} W_{\mathrm{cc} 34} \cos 2 \bar{\alpha}_{2}$

$\chi_{41}^{\prime}=\frac{1}{2} W_{\mathrm{cc} 14} \cos \left(2 \bar{\alpha}_{3}+\bar{\alpha}_{1}+\bar{\alpha}_{2}\right)$

$\chi_{42}^{\prime}=\frac{1}{2} W_{\mathrm{cc} 24} \cos \left(2 \bar{\alpha}_{3}-\bar{\alpha}_{1}+\bar{\alpha}_{2}\right)$

$\chi_{43}^{\prime}=\frac{1}{2} W_{\mathrm{cc} 34} \cos 2 \bar{\alpha}_{2}$

$\chi_{44}^{\prime}=-W_{\mathrm{c} 0}$

$\chi_{11}=\omega_{m} W_{\mathrm{s} 0}$

$\chi_{12}=\omega_{m} W_{\mathrm{cc} 0} \sin 2 \bar{\alpha}_{1}$

$\chi_{13}=\omega_{m} W_{\mathrm{cc} 13} \sin \left(2 \bar{\alpha}_{3}+\bar{\alpha}_{1}-\bar{\alpha}_{2}\right)$

$\chi_{14}=\omega_{m} r_{m} W_{\mathrm{cc} 14} \sin \left(2 \bar{\alpha}_{3}+\bar{\alpha}_{1}+\bar{\alpha}_{2}\right)$

$\chi_{21}=-\omega_{m} W_{\mathrm{cc} 12} \sin 2 \bar{\alpha}_{1}$

$\chi_{22}=\omega_{m} r_{m} W_{\mathrm{s} 0}$

$\chi_{23}=\omega_{m} W_{\mathrm{cc} 23} \sin \left(2 \bar{\alpha}_{3}-\bar{\alpha}_{1}-\bar{\alpha}_{2}\right)$

$\chi_{24}=\omega_{m} W_{\mathrm{cc}} \sin \left(2 \bar{\alpha}_{3}-\bar{\alpha}_{1}+\bar{\alpha}_{2}\right)$

$\chi_{31}=-\omega_{m} W_{\mathrm{cc} 13} \sin \left(2 \bar{\alpha}_{3}+\bar{\alpha}_{1}-\bar{\alpha}_{2}\right)$

$\chi_{32}=-\omega_{m} W_{\mathrm{cc} 23} \sin \left(2 \bar{\alpha}_{3}-\bar{\alpha}_{1}+\bar{\alpha}_{2}\right)$

$\chi_{33}=\omega_{m} W_{\mathrm{s} 0}$ 


$$
\begin{aligned}
& \chi_{34}=\omega_{m} W_{\mathrm{cc} 34} \sin 2 \bar{\alpha}_{2} \\
& \chi_{41}=-\omega_{m} W_{\mathrm{cc} 14} \sin \left(2 \bar{\alpha}_{3}+\bar{\alpha}_{1}+\bar{\alpha}_{2}\right) \\
& \chi_{42}=-\omega_{m} W_{\mathrm{cc} 24} \sin \left(2 \bar{\alpha}_{3}-\bar{\alpha}_{1}+\bar{\alpha}_{2}\right) \\
& \chi_{43}=-\omega_{m} W_{\mathrm{cc} 34} \sin 2 \bar{\alpha}_{2} \\
& \chi_{44}=W_{\mathrm{s} 0} \\
& W_{\mathrm{s} 0}=r_{m}\left[\mu_{x 0}\left(\sin \gamma_{x 0}+\varsigma_{x 0} \varsigma_{\mathrm{e} x} \sin \gamma_{\mathrm{e} x 0}\right)+\mu_{y 0}\left(\sin \gamma_{y 0}+\varsigma_{y 0} \varsigma_{\mathrm{e} y} \sin \gamma_{\mathrm{e} y 0}+\right.\right. \\
& \left.\left.r_{\mathrm{e} 1}^{2} \varsigma_{y 0} \varsigma_{\mathrm{e} \psi 0} \sin \gamma_{\psi y 0}\right)+\mu_{\psi 0}\left(r_{\mathrm{ea}}^{2} \sin \gamma_{\psi 0}+\varsigma_{\mathrm{e} \psi 0} \varsigma_{\psi 0} r_{\mathrm{ea}}^{2} \sin \gamma_{\mathrm{e} \psi 0}\right)\right] \\
& W_{\mathrm{c} 0}=r_{m}\left[\mu_{x 0}\left(\cos \gamma_{x 0}+\varsigma_{x 0} \varsigma_{\mathrm{e} x} \cos \gamma_{\mathrm{e} x 0}\right)+\mu_{y 0}\left(\cos \gamma_{y 0}+\varsigma_{y 0} \varsigma_{\mathrm{e} y} \cos \gamma_{\mathrm{e} y 0}+\right.\right. \\
& \left.\left.r_{\mathrm{e} 1}^{2} \varsigma_{y 0} \varsigma_{\mathrm{e} \psi 0} \cos \gamma_{\psi y 0}\right)+\mu_{\psi 0}\left(r_{\mathrm{ea}}^{2} \cos \gamma_{\psi 0}+\varsigma_{\mathrm{e} \psi 0} \varsigma_{\psi 0} r_{\mathrm{ea}}^{2} \cos \gamma_{\mathrm{e} \psi 0}\right)\right] \\
& W_{\mathrm{cc} 12}=W_{\mathrm{cc} 34}=W_{\mathrm{cc} 0}+r_{m}\left[\mu_{x 0} \varsigma_{x 0} \varsigma_{\mathrm{e} x} \cos \gamma_{\mathrm{e} x 0}-\mu_{y 0}\left(\varsigma_{y 0} \varsigma_{\mathrm{e} y} \cos \gamma_{\mathrm{e} y 0}+\right.\right. \\
& \left.\left.r_{\mathrm{e} 1}^{2} \varsigma_{y 0} \varsigma_{\mathrm{e} \psi 0} \cos \gamma_{\mathrm{e} \psi y 0}\right)+\mu_{\psi 0} r_{\mathrm{ea}}^{2} \varsigma_{\mathrm{e} \psi 0} \varsigma_{\psi 0} \cos \gamma_{\mathrm{e} \psi 0}\right] \\
& W_{\mathrm{cc} 0}=r_{m}\left(\mu_{x 0} \cos \gamma_{x 0}-\mu_{y 0} \cos \gamma_{\mathrm{y} 0}+r_{\mathrm{ea}}^{2} \mu_{\psi 0} \cos \gamma_{\psi 0}\right) \\
& W_{\mathrm{cs} 12}=W_{\mathrm{cs} 34}=W_{\mathrm{cs} 1}+r_{m}\left[-\mu_{x 0} \varsigma_{x 0} \varsigma_{\mathrm{e} x} \sin \gamma_{\mathrm{e} x 0}+\mu_{y 0}\left(\varsigma_{y 0} \varsigma_{\mathrm{e} y} \sin \gamma_{\mathrm{e} y 0}+\right.\right. \\
& \left.\left.r_{\mathrm{e} 1}^{2} \varsigma_{y 0} \varsigma_{\mathrm{e} \psi 0} \sin \gamma_{\mathrm{e} \psi y 0}\right)-\mu_{\psi 0} r_{\mathrm{ea}}^{2} \varsigma_{\mathrm{e} \psi 0} \varsigma_{\psi 0} \sin \gamma_{\mathrm{e} \psi 0}\right] \\
& W_{\mathrm{cs} 1}=r_{m}\left(\mu_{x 0} \cos \gamma_{x 0}-\mu_{y 0} \cos \gamma_{\mathrm{y} 0}+r_{\mathrm{ea}}^{2} \mu_{\psi 0} \cos \gamma_{\psi 0}\right) \\
& W_{\mathrm{cc} 13}=W_{\mathrm{cc} 24}=r_{m}\left[-\mu_{x 0} \varsigma_{x 0} \varsigma_{\mathrm{e} x} \cos \gamma_{\mathrm{e} x 0}-\right. \\
& \left.\mu_{y 0}\left(\varsigma_{y 0} \varsigma_{\mathrm{e} y} \cos \gamma_{\mathrm{e} y 0}-r_{\mathrm{e} 1}^{2} \varsigma_{y 0} \varsigma_{\mathrm{e} \psi 0} \cos \gamma_{\mathrm{e} \psi y 0}\right)-\mu_{\psi 0} \varsigma_{\mathrm{e} \psi 0} \varsigma_{\psi 0} r_{\mathrm{ea}}^{2} \cos \gamma_{\mathrm{e} \psi 0}\right] \\
& W_{\mathrm{cs} 13}=W_{\mathrm{cs} 25}=r_{m}\left[\mu_{x 0} \varsigma_{x 0} \varsigma_{\mathrm{e} x} \sin \gamma_{\mathrm{e} x 0}+\right. \\
& \left.\mu_{y 0}\left(\varsigma_{y 0} \varsigma_{\mathrm{e} y} \sin \gamma_{\mathrm{e} y 0}-r_{\mathrm{e} 1}^{2} \varsigma_{y 0} \varsigma_{\mathrm{e} \psi 0} \sin \gamma_{\mathrm{e} \psi y 0}\right)+\mu_{\psi 0} \varsigma_{\mathrm{e} \psi 0} \varsigma_{\psi 0} r_{\mathrm{ea}}^{2} \sin \gamma_{\mathrm{e} \psi 0}\right] \\
& W_{\mathrm{cc} 14}=W_{\mathrm{cc} 23}=r_{m}\left[\mu_{x 0} \varsigma_{x 0} \varsigma_{\mathrm{e} x} \cos \gamma_{\mathrm{e} x 0}-\right. \\
& \left.\mu_{y 0}\left(\varsigma_{y 0} \varsigma_{\mathrm{e} y} \cos \gamma_{\mathrm{e} y 0}-r_{\mathrm{e} 1}^{2} \varsigma_{y 0} \varsigma_{\mathrm{e} \psi 0} \cos \gamma_{\mathrm{e} \psi y 0}\right)+\mu_{\psi 0} \varsigma_{\mathrm{e} \psi 0} \varsigma_{\psi 0} r_{\mathrm{ea}}^{2} \cos \gamma_{\mathrm{e} \psi 0}\right] \\
& W_{\mathrm{cs} 14}=W_{\mathrm{cs} 23}=r_{m}\left[-\mu_{x 0} \varsigma_{x 0} \varsigma_{\mathrm{e} x} \sin \gamma_{\mathrm{e} x 0}+\right. \\
& \left.\mu_{y 0}\left(\varsigma_{y 0} \varsigma_{\mathrm{e} y} \sin \gamma_{\mathrm{e} y 0}-r_{\mathrm{e} 1}^{2} \varsigma_{y 0} \varsigma_{\mathrm{e} \psi 0} \sin \gamma_{\mathrm{e} \psi y 0}\right)-\mu_{\psi 0} \varsigma_{\mathrm{e} \psi 0} \varsigma_{\psi 0} r_{\mathrm{ea}}^{2} \sin \gamma_{\mathrm{e} \psi 0}\right]
\end{aligned}
$$




\section{Appendix B: Parameters of the vibrating system}

Table A1

Parameters of the four induction motors

\begin{tabular}{lllll}
\hline Parameters & Motor 11 & Motor 12 & Motor 21 & Motor 22 \\
\hline Rated power $(\mathrm{kW})$ & 3.7 & 0.75 & 3.7 & 0.75 \\
Poles & 6 & 6 & 6 & 6 \\
Rated frequency $(\mathrm{Hz})$ & 50 & 50 & 50 & 50 \\
Rated voltage $(\mathrm{V})$ & 220 & 220 & 220 & 220 \\
Rated rotational speed (r/min) & 980 & 980 & 980 & 980 \\
Stator resistance $(\Omega)$ & 0.54 & 3.35 & 0.54 & 3.35 \\
Rotor resistance referred to stator $(\Omega)$ & 0.56 & 3.40 & 0.56 & 3.40 \\
Stator inductance $(\mathrm{H})$ & 0.141 & 0.170 & 0.141 & 0.170 \\
Rotor inductance referred to stator $(\mathrm{H})$ & 0.143 & 0.170 & 0.143 & 0.170 \\
Mutual inductance $(\mathrm{H})$ & 0.138 & 0.164 & 0.138 & 0.164 \\
Rotor damping coefficient $(\mathrm{N} . \mathrm{m} . \mathrm{s} / \mathrm{rad})$ & 0.02 & 0.005 & 0.04 & 0.005 \\
\hline
\end{tabular}

Table A2

Parameters of the vibrating system

\begin{tabular}{llll}
\hline Parameters & V1 & V2 & Isolation frame \\
\hline Mass of the vibrating frame $(\mathrm{kg})$ & 2400 & 2400 & 1000 \\
Moment of inertia about its centriod $\left(\mathrm{kg} \cdot \mathrm{m}^{2}\right)$ & 2190 & 2190 & 1600 \\
Mass of the eccentric lump $(\mathrm{kg})$ & 30 & 30 & \\
Eccentric radius $(\mathrm{m})$ & 0.2 & 0.2 & \\
Stiffness of springs in $x$-direction $(\mathrm{N} / \mathrm{m})$ & 630000 & 630000 & 800000 \\
Stiffness of springs in $y$-direction $(\mathrm{N} / \mathrm{m})$ & 630000 & 630000 & 800000 \\
Stiffness of springs in $\psi$-direction $(\mathrm{N} \cdot \mathrm{m} / \mathrm{rad})$ & 550000 & 550000 & 600000 \\
Damping constant in $x$-direction $(\mathrm{N} /(\mathrm{m} / \mathrm{s}))$ & 3850 & 3850 & 18000 \\
Damping constant in $y$-direction $(\mathrm{N} /(\mathrm{m} / \mathrm{s}))$ & 3850 & 3850 & 18000 \\
Damping constant in $\psi$-direction $(\mathrm{N} \cdot \mathrm{m} /(\mathrm{rad} / \mathrm{s}))$ & 3400 & 3400 & 20000 \\
\hline
\end{tabular}

\section{References}

[1] J.M. Balthaza, J.L. Palacios Felix and MLRF B. Reyolando, Some comments on the numerical simulation of self-synchronization of four non-ideal exciters, Applied Mathematics and Computation 164 (2005), 615-625.

[2] I.I. Blekham, Synchronization in Science and Technology, ASME Press, New York, 1988.

[3] I.I. Blekhman, Synchronization of Dynamical Systems, Science, Moscow, 1971, (in Russian).

[4] I.I. Blekhman, Vibrational Mechanics, World Scientific, Singapore, 2000.

[5] I.I. Blekhman, Selected Topics in Vibrational Mechanics, World Scientific, Singapore, 2004.

[6] I.I. Blekhman, A.L. Fradkov, H. Nijmeijier et al., On self-synchronization and controlled synchronization, System \& Control Letters 31 (1997), 299-305.

[7] I.I. Blekhman, A.L. Fradkov, O.P. Tomchina et al., Self-synchronization and controlled synchronization, Mathematics and Computers in Simulation 58 (2002), 367-384.

[8] I.I. Blekhman and N.P. Yaroshevich, Extension of the domain of applicability of the integral stability criterion (extremum property) in synchronization problems, Journal of Applied Mathematics and Mechanics 68 (2004), 839-846.

[9] K. Czolczynski, P. Perlikowski, A. Stefański et al., Synchronization of self-excited oscillators suspended on elastic structure, Chaos, Solitons and Fractals 32 (2007), 937-943.

[10] A.L. Fradkov and B. Andrievsky, Synchronization and relations in the motion of two-pendulum system, International Journal of Non-Linear Mechanics 42 (2007), 895-901.

[11] Q.S. Lu, Qualitative Methods and Bifurcations of Ordinary Differential Equation, Press of Beijing University of Aeronautics and Astronautics, Beijing, 1989, (in Chinese).

[12] M. Senator, Synchronization of two coupled escapement-driven pendulum clocks, Journal of Sound and Vibration 291 (2006), 566-603.

[13] H.A. Tanaka, A.J. Lichtenberg and S. Oishi, Self-synchronization of coupled oscillators with hysteretic responses, Physica D 100 (1997), 279-300.

[14] B.C. Wen and F.Q. Liu, Theory of Vibrating Machines and Its Applications, Machine Industry Press, Beijing, 1982, (in Chinese).

[15] B.C. Wen, J. Fan, C.Y. Zhao et al., Vibratory Synchronization and Controlled Synchronization in Engineering, Science Press, Beijing, 2009. 
[16] R. Yamapi and P. Woafo, Synchronized states in a ring of four mutually coupled self-sustained electromechanical devices, Communications in Nonlinear Science and Numerical Simulation 11 (2006), 186-202.

[17] R. Yamapi and P. Woafo, Dynamics and synchronization of coupled self-sustained electromechanical devices, Journal of Sound and Vibration 285 (2005), 1151-1171.

[18] C.Y. Zhao, H.T. Zhu, R.Z. Wang et al., Synchronization of two-identical coupled exciters in a non-resonant vibrating system of linear motion. Part I: Theoretical analysis, Shock and Vibration 16 (2009), 505-516.

[19] C.Y. Zhao, H.T. Zhu, T.J. Bai et al., Synchronization of two-identical coupled exciters in a non-resonant vibrating system of linear motion. Part II: Numeric analysis, Shock and Vibration 16 (2009), 517-528.

[20] C.Y. Zhao, H.T. Zhu, Y.M. Zhang et al., Synchronization of two coupled exciters in a vibrating system of spatial motion, Acta Mech Sin, DOI 10.1007/s10409-009-0311-1.

[21] C.Y. Zhao, Y.M. Zhang and B.C. Wen, Synchronization and General Dynamic Symmetry of a Vibrating System with Two Exciters Rotating in Opposite Directions, Chinese Physics B 19 (2010), 030301.

[22] X.H. Zhang and Q.L. Zhang, Control Theory of Nonlinear Differential Algebraic System and its Applications, Science Press, Beijing, 2007, (in Chinese). 

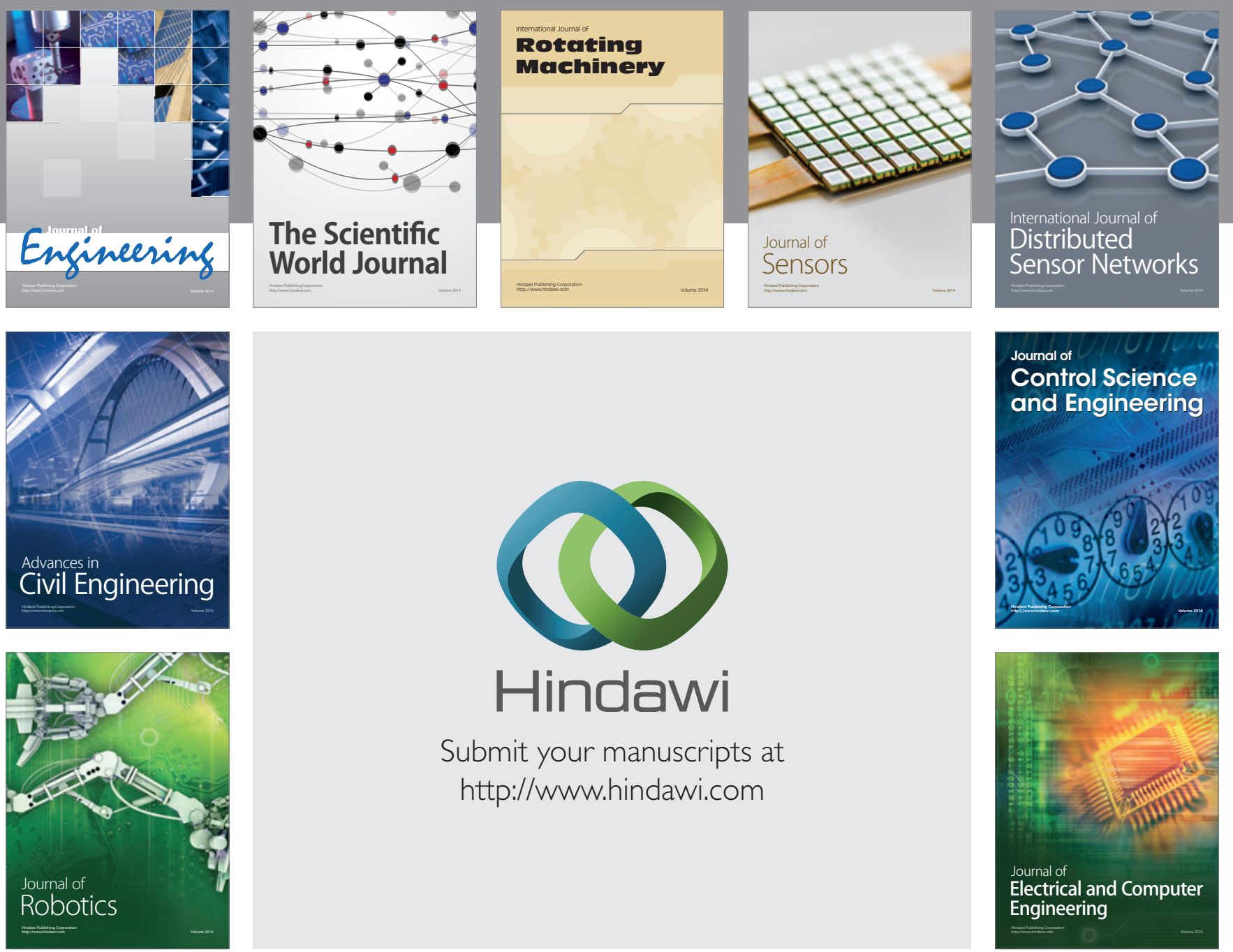

Submit your manuscripts at

http://www.hindawi.com
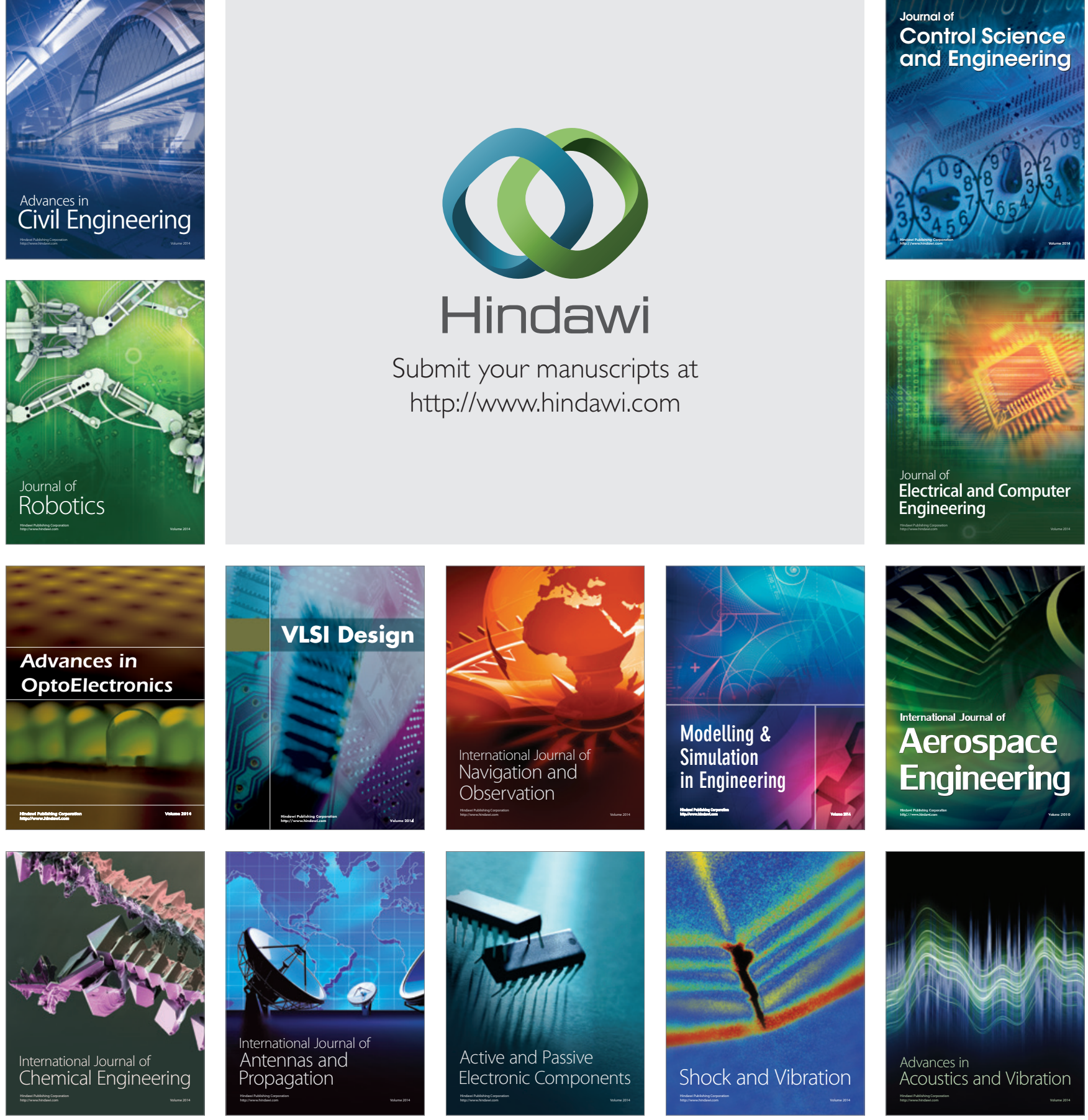